\title{
Skew-selfadjoint Dirac systems with rational rectangular Weyl functions: explicit solutions of direct and inverse problems and integrable wave equations
}

\author{
B. Fritzsche, M.A. Kaashoek, B. Kirstein, A.L. Sakhnovich
}

\begin{abstract}
In this paper we study direct and inverse problems for discrete and continuous time skew-selfadjoint Dirac systems with rectangular (possibly non-square) pseudo-exponential potentials. For such a system the Weyl function is a strictly proper rational rectangular matrix function and any strictly proper rational matrix function appears in this way. In fact, extending earlier results, given a strictly proper rational matrix function we present an explicit procedure to recover the corresponding potential using techniques from mathematical system and control theory. We also introduce and study a nonlinear generalized discrete Heisenberg magnet model, extending earlier results for the isotropic case. A large part of the paper is devoted to the related discrete time systems of which the pseudo-exponential potential depends on an additional continuous time parameter. Our techniques allows us to obtain explicit solutions for the generalized discrete Heisenberg magnet model and evolution of the Weyl functions.
\end{abstract}

$\operatorname{MSC}(2010)$ :

Keywords: Weyl function, Weyl theory, continuous Dirac system, discrete Dirac system, rectangular matrix potential, pseudo-exponential potential, direct problem, inverse problem, explicit solution, rational matrix function, realization, generalized discrete Heisenberg magnet model. 


\section{Introduction}

A skew-selfadjoint Dirac system (also called a pseudo-canonical, ZakharovShabat or AKNS system) has the form:

$$
\begin{aligned}
\frac{d}{d x} y(x, z) & =(i z j+j V(x)) y(x, z) \quad\left(x \in \mathbb{R}_{+}, \quad z \in \mathbb{C}\right), \\
j & =\left[\begin{array}{cc}
I_{m_{1}} & 0 \\
0 & -I_{m_{2}}
\end{array}\right], \quad V=\left[\begin{array}{cc}
0 & v \\
v^{*} & 0
\end{array}\right],
\end{aligned}
$$

where $\mathbb{C}$ stands for the complex plane, $\mathbb{R}_{+}$denotes the non-negative real semi-axis, $I_{m_{k}}$ is the $m_{k} \times m_{k}$ identity matrix, $v(x)$ is an $m_{1} \times m_{2}$ matrix function, which is called the potential of the system, and $j$ and $V(x)$ are $m \times m$ matrices, $m:=m_{1}+m_{2}$. Note that $(j V)^{*}=-j V$, and therefore the system (1.1) is called skew-selfadjoint.

Like a selfadjoint Dirac system $\frac{d}{d x} y(x, z)=i(z j+j V(x)) y(x, z)$, the system (1.1) is also an auxiliary system for various important integrable nonlinear wave equations and the case $m_{1} \neq m_{2}$ corresponds to rectangular and multicomponent versions of these equations. Here we solve explicitly (in terms of Weyl functions) direct and inverse problems for the system (1.1) for the case when the rectangular (possibly non-square) potential $v$ is pseudoexponential (see formula (2.1)). The direct problem consists in constructing the Weyl function and inverse problem is the problem to recover $v$ from the Weyl function.

We also derive explicit solutions of direct and inverse problems for a discrete analogue of the skew-selfadjoint system (1.1), namely, for the system

$$
\begin{aligned}
y_{k+1}(z)=\left(I_{m}+i z^{-1} C_{k}\right) y_{k}(z), & C_{k}=U_{k}^{*} j U_{k}, \text { where } \\
U_{k}^{*} U_{k} & =U_{k} U_{k}^{*}=I_{m}, \quad k=0,1,2, \ldots .
\end{aligned}
$$

This system was studied in [27] for the important subcase $m_{1}=m_{2}$ and explicit solutions of direct and inverse problems were obtained for that subcase. See [18] for the discrete analogue of selfadjoint Dirac system (and its general Weyl theory).

A large part of the paper is devoted to the case when the system (1.3) depends on an additional continuous time parameter. A special choice of the

additional parameter allows us to introduce a generalized discrete Heisenberg magnet model. We use our results on system (1.3) with a general-type $j$ in 
order to construct explicit solutions and the evolution of the Weyl function for this generalized model. The results obtained generalize those for the case $m_{1}=m_{2}=1$ in [27] which dealt with the well-known discrete isotropic Heisenberg magnet model [14,47].

History. Direct and inverse spectral problems for Dirac systems have a long and interesting history. For the selfadjoint case with a scalar continuous potential $v$ it all started with the seminal paper [30] by M.G. Krein (see also the discussions in $[3,45])$. This theory is closely related to the Weyl theory (see, e.g., $[3,8,34,39,41,44]$ and references therein). The Weyl theory for the skew-selfadjoint systems of type (1.1) was developed much later [8,16, 23, 35, $40,41]$ and the method of operator identities [42-44] played a fundamental role in these studies.

Particular subclasses of general-type potentials $v$ are of special interest, since, for some subclasses, direct and inverse problems may be solved explicitly. Explicit solutions of spectral problems for selfadjoint Dirac systems with the strictly pseudo-exponential square potentials $v$ were given in $[1,2]$. These constructions were based on the procedure to solve direct and inverse problems for systems with general-type potentials. A few years later direct and inverse problems in terms of spectral and Weyl functions were solved explicitly in [22] for a wider class of selfadjoint Dirac systems, that is, for systems with pseudo-exponential square potentials $v$ (see also [24]). The same problem for system (1.1) was dealt with in [23]. Moreover, the above mentioned problems for systems with pseudo-exponential potentials were studied directly instead of using procedures for general-type selfadjoint (or, correspondingly, skew-selfadjoint) Dirac systems as in $[1,2]$. We note that direct methods in explicit solution of spectral problems go back to the works by Bäcklund [4] and Darboux [10,11] with essential further developments in $[9,12,19,20,31]$. Finally, direct and inverse problems for selfadjoint Dirac systems with the pseudo-exponential rectangular (possibly non-square) potentials $v$ were solved explicitly in terms of Weyl functions in [17].

Contents. The paper consists of four sections (the present introduction included) and an appendix. Section 2 deals with direct and inverse problem for continuous time systems. In this section we develop further the results from [23] on skew-selfadjoint system (1.2) with square potentials, also using some ideas and results from the works $[16,17]$ on systems with general-type rectangular (non-square) potentials. Section 3 treats direct and inverse problems for discrete time systems, generalizing earlier results from [27] to the 
rectangular (non-square) case. Finally, Section 4 is devoted to generalized discrete Heisenberg magnet model, which is equivalent to the compatibility condition of two systems depending on two parameters $t$ and $k$ where $t$ is non-negative real and $k \in \mathbb{N}_{0}$. For the convenience of the reader the appendix Section A presents a number of general facts regarding admissible quadruples that are used throughout the paper. This theory is closely related to the triples approach [22-24,27] and generalized Bäcklund-Darboux transformation (GBDT) method $[15,17,37,38,41]$ (see also references therein).

Notation. We conclude with some information on notations that are used throughout the paper. As usual $\mathbb{N}_{0}$ stands for the set of non-negative integers, i.e., the natural numbers with zero included, and $\mathbb{R}_{+}$denotes the set of nonnegative real values. The symbols $\mathbb{C}_{+}$and $\mathbb{C}_{-}$stand for the upper and lower half-plane, respectively. Furthermore, $\overline{\mathbb{C}_{+}}$denotes the closed upper half-plane (i.e., $\mathbb{C}_{+} \cup \mathbb{R}$ ), and $\mathbb{C}_{M}$ stands for the open half-plane $\{z: \Im(z)>M>0\}$. By $\|\cdot\|$ we denote the $\ell^{2}$ vector norm or the induced matrix norm, Span stands for the linear span, and $\sigma(\alpha)$ stands for the spectrum of $\alpha$. The class of $m_{2} \times m_{1}$ contractive matrix functions (Schur matrix functions) on some domain $\Omega$ is denoted by $\mathcal{S}_{m_{2} \times m_{1}}(\Omega)$. We write $S>0$ when the matrix $S$ is positive definite. The matrix $\left(\alpha^{-1}\right)^{*}$ is denoted by $\alpha^{-*}$ and $\operatorname{Im} \alpha$ stands for the image of $\alpha$.

\section{Continuous case: direct and inverse problem}

We begin with introducing the notion of a pseudo-exponential potential. The starting point is a matrix function $v$ of the form

$$
v(x)=2 \vartheta_{1}^{*} e^{i x \alpha^{*}} S(x)^{-1} e^{i x \alpha} \vartheta_{2}, \quad x \in \mathbb{R}_{+} .
$$

Here $\vartheta_{1}$ and $\vartheta_{2}$ are matrices of sizes $n \times m_{1}$ and $n \times m_{2}$, respectively, $\alpha$ is an $n \times n$ matrix, and $S$ is the $n \times n$ matrix function given by

$$
S(x)=S_{0}+\int_{0}^{x} \Lambda(t) j \Lambda(t)^{*} d t, \quad S_{0}>0, \quad \Lambda(x):=\left[\begin{array}{ll}
e^{-i x \alpha} \vartheta_{1} & e^{i x \alpha} \vartheta_{2}
\end{array}\right] .
$$

Furthermore, we require the initial value $S_{0}$ in (2.2) to satisfy the following matrix identity:

$$
\alpha S_{0}-S_{0} \alpha^{*}=i\left(\vartheta_{1} \vartheta_{1}^{*}+\vartheta_{2} \vartheta_{2}^{*}\right)
$$


From (2.1) and (2.2) it is clear that the potential $v$ in (2.1) is uniquely determined by the quadruple $\left\{\alpha, S_{0}, \vartheta_{1}, \vartheta_{2}\right\}$. In combination with $S_{0}$ being positive definite, the identity (2.3) implies that $S(x)$ is also positive definite; see (2.8) below. In particular, $S(x)$ is invertible for each $x \geq 0$, and hence $v$ is well-defined.

When $S_{0}>0$ and (2.3) holds we call the quadruple $\left\{\alpha, S_{0}, \vartheta_{1}, \vartheta_{2}\right\}$ an admissible quadruple. We call $v$ in (2.1) the pseudo-exponential potential generated by the admissible quadruple $\left\{\alpha, S_{0}, \vartheta_{1}, \vartheta_{2}\right\}$. See appendix Section $\mathrm{A}$ for a brief review of properties of an admissible quadruple and the relation with the theory of S-nodes.

The definition of a pseudo-exponential potential given here is somewhat different from the definition in [23] which starts with [23, Eq. (0.2)]. However, Proposition 1.1 in [23] tells us that a pseudo-exponential potential in sense of [23] is also a pseudo-exponential potential as defined above. With some minor modifications the reverse implication is also true (see Proposition 2.8 at the end of this section).

In the present paper, as opposed to [23], we do not require the matrices $\vartheta_{1}$ and $\vartheta_{2}$ to be square, i.e., $m_{1}$ and $m_{2}$ are not required to be equal. Note that in [23] the matrix $S_{0}$ is just the $n \times n$ identity matrix but, as the next lemma shows, it is convenient to allow $S_{0}$ to be just positive definite.

Lemma 2.1. Let $\left\{\alpha, S_{0}, \vartheta_{1}, \vartheta_{2}\right\}$ be an admissible quadruple, and for each $x \in \mathbb{R}_{+}$let $\Sigma(x)$ be the quadruple defined by

$$
\Sigma(x)=\left\{\alpha, S(x), e^{-i x \alpha} \vartheta_{1}, e^{i x \alpha} \vartheta_{2}\right\}
$$

where $S(x)$ is the matrix defined by the first identity in (2.2). Then $\Sigma(x)$ is an admissible quadruple for each $x \in \mathbb{R}_{+}$.

Proof. We first show that

$$
\alpha S(x)-S(x) \alpha^{*}=i \Lambda(x) \Lambda(x)^{*}, \quad x \in \mathbb{R}_{+} .
$$

To prove this identity note that the relations in (2.2) are equivalent to

$$
\begin{aligned}
& \frac{d}{d x} \Lambda(x)=-i \alpha \Lambda(x) j, \quad \Lambda(0)=\left[\begin{array}{ll}
\vartheta_{1} & \vartheta_{2}
\end{array}\right] \quad\left(x \in \mathbb{R}_{+}\right) \\
& \frac{d}{d x} S(x)=\Lambda(x) j \Lambda(x)^{*}, \quad S(0)=S_{0} \quad\left(x \in \mathbb{R}_{+}\right) .
\end{aligned}
$$


Here $j$ is the signature matrix defined (1.2). If follows that

$$
\begin{aligned}
& \frac{d}{d x}\left(\alpha S(x)-S(x) \alpha^{*}\right)=\alpha \Lambda(x) j \Lambda(x)^{*}-\Lambda(x) j \Lambda(x)^{*} \alpha^{*}, \\
& \frac{d}{d x} i \Lambda(x) \Lambda(x)^{*}=\alpha \Lambda(x) j \Lambda(x)^{*}-\Lambda(x) j \Lambda(x)^{*} \alpha^{*} .
\end{aligned}
$$

On the other hand, using (2.3), the functions $\alpha S(x)-S(x) \alpha^{*}$ and $i \Lambda(x) \Lambda(x)^{*}$ have the same value at $x=0$. But then (2.5) holds true.

It remains to show that $S(x)$ is positive definite for each $x \geq 0$. To do this we prove the following inequality:

$$
e^{-i x \alpha} S(x) e^{i x \alpha^{*}} \geq S_{0}, \quad x \in \mathbb{R}_{+} .
$$

Using (2.7) and (2.5) we have

$$
\begin{aligned}
\frac{d}{d x}\left(e^{-i x \alpha} S(x) e^{i x \alpha^{*}}\right) & =e^{-i x \alpha}\left(\frac{d}{d x} S(x)\right) e^{i x \alpha^{*}} \\
& -i e^{-i x \alpha}\left(\alpha S(x)-S(x) \alpha^{*}\right) e^{i x \alpha^{*}} \\
& =\Lambda(x) j \Lambda(x)^{*}+\Lambda(x) \Lambda(x)^{*} \\
& =2 e^{-i x \alpha} \vartheta_{1}^{*} \vartheta_{1} e^{i x \alpha^{*}} \geq 0, \quad x \in \mathbb{R}_{+} .
\end{aligned}
$$

But then

$$
e^{-i x \alpha} S(x) e^{i x \alpha^{*}}-S_{0}=\int_{0}^{x} \frac{d}{d t}\left(e^{-i t \alpha} S(t) e^{i t \alpha^{*}}\right) \geq 0, \quad x \in \mathbb{R}_{+} .
$$

In particular, (2.8) holds true.

We note that the quadruple $\left\{\alpha, S_{0}, \vartheta_{1}, \vartheta_{2}\right\}$ generating $v$ coincides with $\Sigma(0)$ in $(2.4)$.

Using straightforward modifications of the proof of [23, Theorem 1.2] (or particular cases of the more general [37, Theorem 3] or [38, Theorem 1.2 and Proposition 1.4]) we obtain the next proposition.

Proposition 2.2. Let $v$ be the pseudo-exponential potential generated by the admissible quadruple $\Sigma(0)=\left\{\alpha, S_{0}, \vartheta_{1}, \vartheta_{2}\right\}$, and let $\Sigma(x)$ be the admissible quadruple defined by (2.4). Then the fundamental solution $u$ of the system (1.1), normalized by $u(0, z) \equiv I_{m}$, where $m=m_{1}+m_{2}$, admits the following representation

$$
\begin{aligned}
& u(x, z)=W_{\Sigma(x)}(z) e^{i x z j} W_{\Sigma(0)}(z)^{-1}, \\
& W_{\Sigma(x)}(z):=I_{m}+i \Lambda(x)^{*} S(x)^{-1}\left(z I_{n}-\alpha\right)^{-1} \Lambda(x) .
\end{aligned}
$$


The transfer function of the form (2.10) (the transfer function in Lev Sakhnovich form) was introduced and studied in [42] (see also [41,43,44] and references therein). In our case, we refer to $W_{\Sigma(x)}$ as the the transfer function associated with the admissible quadruple $\Sigma(x)$; see the first paragraph of Section A. The next result is a simple generalization of [23, Proposition 1.4].

Proposition 2.3. Pseudo-exponential potentials $v$ are bounded on the semiaxis $x \geq 0$.

Proof. Assume that the potential $v$ is generated by the admissible quadruple $\Sigma(0)=\left\{\alpha, S_{0}, \vartheta_{1}, \vartheta_{2}\right\}$, and let $\Sigma(x)$ be the admissible quadruple defined by (2.4). From Lemma A.1 we know that the eigenvalues of $\alpha$ belong to $\overline{\mathbb{C}_{+}}$. Furthermore, using the identity (A.9) with $\Sigma(x)$ in place of $\Sigma$, we see that

$$
i(z-\bar{z}) \Lambda(x)^{*}\left(\bar{z} I_{n}-\alpha^{*}\right)^{-1} S(x)^{-1}\left(z I_{n}-\alpha\right)^{-1} \Lambda(x) \leq I_{m}, \quad z \in \mathbb{C}_{-} .
$$

Since the resolvent $\left(z I_{n}-\alpha\right)^{-1}$ is well-defined in any open domain $\Omega$ in $\mathbb{C}_{-}$, we have $\operatorname{Span}_{z \in \Omega}\left(z I_{n}-\alpha\right)^{-1} \vartheta_{k} \supseteq \vartheta_{k}$ for $k=1,2$. Therefore, inequality $(2.11)$ (where $\Lambda(x)$ is given by (2.2)) implies that

$$
\sup _{x \geq 0}\left(\left\|S(x)^{-1 / 2} e^{-i x \alpha} \vartheta_{1}\right\|+\left\|S(x)^{-1 / 2} e^{i x \alpha} \vartheta_{2}\right\|\right)<\infty .
$$

From (2.1) and (2.12), it is immediate that $v$ is bounded, that is, for some $M>0$ we have

$$
\|v(x)\| \leq M \quad x \in \mathbb{R}_{+},
$$

which completes the proof.

Weyl function: the direct problem. The concept of a Weyl function of Dirac system has a long history (see the Introduction). Following the definition of a Weyl function for Dirac systems with square potentials (see also [41] for the case of non-square potentials) we say that a meromorphic function $\varphi$ satisfying (2.13) is a Weyl function of the system (1.1) whenever it satisfies the inequality

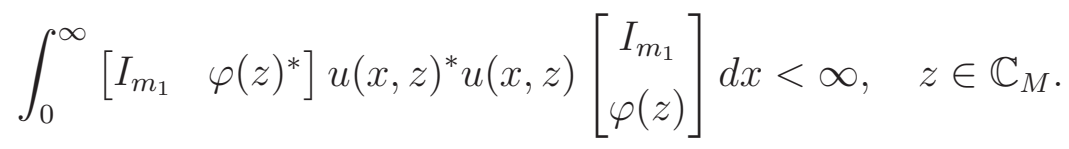


Here $u$ is the fundamental solution of $(1.1)$ normalized by $u(0, z)=I_{m}$. We note that for skew-selfadjoint Dirac systems (1.1) Weyl functions have been introduced in [16] in an equivalent but different way. However, Proposition 2.2 and Corollary 2.8 from [16] immediately yield the following result concerning direct problem.

Proposition 2.4. Let (2.13) hold. Then there is a unique function $\varphi$ such that (2.14) is valid. This function $\varphi$ is analytic and contractive in $\mathbb{C}_{M}$ (i.e., $\left.\varphi \in \mathcal{S}_{m_{2} \times m_{1}}\left(\mathbb{C}_{M}\right)\right)$.

For the case of a pseudo-exponential potential $v$ we produce an explicit expression for the Weyl function.

Theorem 2.5. Consider the skew-selfadjoint Dirac system (1.1), and assume that $v$ is a pseudo-exponential potential generated by the admissible quadruple $\Sigma(0)=\left\{\alpha, S_{0}, \vartheta_{1}, \vartheta_{2}\right\}$. Then the Weyl function $\varphi$ of the Dirac system (1.1) is given by

$$
\varphi(z)=i \vartheta_{2}^{*} S_{0}^{-1}\left(z I_{n}-\alpha^{\times}\right)^{-1} \vartheta_{1}, \quad \alpha^{\times}:=\alpha-i \vartheta_{1} \vartheta_{1}^{*} S_{0}^{-1} .
$$

Proof. From the proof of Lemma A.2 we know that (2.15) can be rewritten in the form:

$$
\varphi(z)=i \vartheta_{2}^{*} S_{0}^{-1}\left(z I_{n}-\alpha\right)^{-1} \vartheta_{1}\left(I_{m_{1}}+i \vartheta_{1}^{*} S_{0}^{-1}\left(z I_{n}-\alpha\right)^{-1} \vartheta_{1}\right)^{-1} .
$$

Taking into account the equivalence of (2.15) and (2.16) and using (2.10) together with the second equality in $(2.2)$ (both at $x=0$ ), we derive that $\varphi$ of the form (2.15) satisfies the relation:

$$
\left[\begin{array}{c}
I_{m_{1}} \\
\varphi(z)
\end{array}\right]=W_{\Sigma(0)}(z)\left[\begin{array}{c}
I_{m_{1}} \\
0
\end{array}\right]\left(I_{m_{1}}+i \vartheta_{1}^{*} S_{0}^{-1}\left(z I_{n}-\alpha\right)^{-1} \vartheta_{1}\right)^{-1} .
$$

Formulas (2.9) and (2.17) imply that

$$
u(x, z)\left[\begin{array}{c}
I_{m_{1}} \\
\varphi(z)
\end{array}\right]=e^{i x z} W_{\Sigma(x)}(z)\left[\begin{array}{c}
I_{m_{1}} \\
0
\end{array}\right]\left(I_{m_{1}}+i \vartheta_{1}^{*} S_{0}^{-1}\left(z I_{n}-\alpha\right)^{-1} \vartheta_{1}\right)^{-1}
$$

Using $S_{0}>0$ and taking inverses in (2.8), we see that

$$
\sup _{x \geq 0}\left\|e^{-i x \alpha^{*}} S(x)^{-1} e^{i x \alpha}\right\|<\infty .
$$


This yields the inequality

$$
\sup _{x \geq 0}\left\|e^{-\eta x} S(x)^{-1}\right\|<\infty \text { for sufficiently large values of } \eta \text {. }
$$

From (2.19) and the identity (A.9) with $\Sigma(x)$ in place of $\Sigma$, it then follows that the function $x \rightarrow e^{i(z-\bar{z}) x} W_{\Sigma(x)}(z)^{*} W_{\Sigma(x)}(z)$ belongs to $L_{m \times m}^{2}(0, \infty)$ for sufficiently large values of $\Im(z)$ :

$$
e^{i(z-\bar{z}) x} W_{\Sigma(x)}(z)^{*} W_{\Sigma(x)}(z) \in L_{m \times m}^{2}(0, \infty) .
$$

That is, the entries of $e^{i(z-\bar{z}) x} W_{\Sigma(x)}(z)^{*} W_{\Sigma(x)}(z)$ are squarely summable with respect to $x$ for sufficiently large values of $\Im(z)$. Finally, in view of (2.18) and (2.20), the inequality (2.14) holds for $\varphi$ given by (2.15) and for sufficiently large values of $\Im(z)$.

Taking into account Proposition 2.4 and the analiticity of $\varphi$ given by (2.15), we see that (2.14) holds for all $z \in\left(\mathbb{C}_{M} \backslash \sigma\left(\alpha^{\times}\right)\right)$, that is, $\varphi$ is the Weyl function.

Weyl function: the inverse problem. Theorem 2.5 presents the solution for the direct problem. We now turn to the inverse problem. The uniqueness theorem below is immediate from [41, Theorem 3.21 and Corollary 3.25] (see also [16]).

Theorem 2.6. Let $\varphi$ be a Weyl function of a skew-selfadjoint Dirac system with a potential $v$ which is bounded on $[0, \infty)$. Then this $v$ can be uniquely recovered from $\varphi$.

For the case of pseudo-exponential potentials we have an explicit procedure to recover the potential from the Weyl function. This procedure uses such well-known notions from control theory as realization, minimal realization and McMillan degree (see, e.g., [5,28] or [41, App. B]). We note that, according to Theorem 2.5, the Weyl function $\varphi$ of a skew-selfadjoint Dirac system with a pseudo-exponential potential is a strictly proper rational matrix function.

Theorem 2.7. Let $\varphi$ be a strictly proper rational $m_{2} \times m_{1}$ matrix function. Then $\varphi$ is the Weyl function of a skew-selfadjoint Dirac system with a pseudo-exponential potential $v$ generated by an admissible quadruple. The 
corresponding quadruple $\left\{\alpha, S_{0}, \vartheta_{1}, \vartheta_{2}\right\}$ can be obtained explicitly by using the following procedure. First, we construct a minimal realization of $\varphi$ :

$$
\varphi(z)=i \theta_{2}^{*}\left(z I_{n}-\gamma\right)^{-1} \theta_{1} .
$$

Next, we choose $X$ to be the unique positive definite solution $X$ of the Riccati equation $\gamma X-X \gamma^{*}-i X \theta_{2} \theta_{2}^{*} X+i \theta_{1} \theta_{1}^{*}=0$. Finally, we put

$$
S_{0}=I_{n}, \quad \vartheta_{1}=X^{-1 / 2} \theta_{1}, \quad \vartheta_{2}=X^{1 / 2} \theta_{2}, \quad \alpha=X^{-1 / 2} \gamma X^{1 / 2}+i \vartheta_{1} \vartheta_{1}^{*} .
$$

Proof. Given a strictly proper rational matrix function $\varphi$, Theorem A.3 provides the described above three step procedure to construct a quadruple $\left\{\alpha, S_{0}, \vartheta_{1}, \vartheta_{2}\right\}$ such that representation (2.15) of $\varphi$ holds. It also follows from Theorem A.3 that the procedure is well-defined and the quadruple $\left\{\alpha, S_{0}, \vartheta_{1}, \vartheta_{2}\right\}$ is admissible. Then we know from Theorem 2.5 that $\varphi$ we started with is precisely the Weyl function of the skew-selfadjoint Dirac system with the potential $v$ generated by $\left\{\alpha, S_{0}, \vartheta_{1}, \vartheta_{2}\right\}$.

As we note in the second paragraph before Lemma 2.1 the definition of a pseudo-exponential potential given in the beginning of this section differs from the definition employed in [23] which starts from formula (0.2) in [23]. The next proposition presents the analogue of formula (0.2) in [23], which coincides with $(0.2)$ for the case $m_{1}=m_{2}$ and $S_{0}=I_{n}$. The conclusion is that the two definitions lead to the same class of potentials.

Proposition 2.8. Let $v$ be the pseudo-exponential potential generated by the admissible quadruple $\left\{\alpha, S_{0}, \vartheta_{1}, \vartheta_{2}\right\}$, and let $A$ be the $2 n \times 2 n$ matrix defined by

$$
A=\left[\begin{array}{cc}
\alpha^{*} & 0 \\
-\vartheta_{1} \vartheta_{1}^{*} & \alpha
\end{array}\right]
$$

Then the potential $v$ is also given by

$$
v(x)=2 \vartheta_{1}^{*}\left(\left[\begin{array}{ll}
S_{0} & -i I_{n}
\end{array}\right] e^{-2 i x A}\left[\begin{array}{c}
I_{n} \\
0
\end{array}\right]\right)^{-1} \vartheta_{2}, \quad x \in \mathbb{R}_{+} .
$$

The proof of the proposition is given in Appendix (and is close to the considerations in [23]). 


\section{Discrete case: direct and inverse problem}

Recall that the discrete skew-selfadjoint Dirac system (SkDDS) has the form:

$$
y_{k+1}(z)=\left(I_{m}+i z^{-1} C_{k}\right) y_{k}(z), \quad C_{k}=U_{k}^{*} j U_{k} \quad\left(k \in \mathbb{N}_{0}\right) .
$$

Here $\mathbb{N}_{0}$ stands for the set of non-negative integers, the matrices $U_{k}$ are unitary of size $m \times m$, where $m$ does not depent on $k$, and $j$ is the $m \times m$ signature matrix defined by (1.2) with $m=m_{1}+m_{2}$ and with $m_{1}$ and $m_{2}$ not depending on $k$. The sequence $\left\{C_{k}\right\}_{k \in \mathbb{N}_{0}}$ is called the potential of the system. Note that the second part of (3.1) implies that $C_{k}=C_{k}^{*}=C_{k}^{-1}$ for each $k \in \mathbb{N}_{0}$.

Definition 3.1. The Weyl function of SkDDS is an $m_{1} \times m_{2}$ meromorphic matrix function $\varphi(z)$ on $\mathbb{C}_{M}$ (for some $M>0$ ), which satisfies the inequality

$$
\sum_{k=0}^{\infty}\left[\varphi(z)^{*} \quad I_{m_{2}}\right] w_{k}(z)^{*} w_{k}(z)\left[\begin{array}{c}
\varphi(z) \\
I_{m_{2}}
\end{array}\right]<\infty
$$

where $w_{k}(z)$ is the fundamental solution of SkDDS normalized by $w_{0}(z) \equiv I_{m}$.

We shall consider the case when the potentials $\left\{C_{k}\right\}_{k \in \mathbb{N}_{0}}$ are pseudoexponential (see Definition 3.3 below), and we shall show that for such potentials the Weyl function can be constructed explicitly.

Pseudo-exponential potentials have been introduced in [27] for the square case when $m_{1}=m_{2}$. We shall show that the same scheme developed in [27] for constructing the corresponding Weyl function also works in the nonsquare case when $m_{1}$ and $m_{2}$ are not equal.

Similar to the continuous case, our starting point to define pseudo-exponential potentials is an admissible quadruple $\Sigma_{0}=\left\{\alpha, S_{0}, \vartheta_{1}, \vartheta_{2}\right\}$, and $\Lambda_{0}=$ $\left[\begin{array}{ll}\vartheta_{1} & \vartheta_{2}\end{array}\right]$. In addition, we assume that $\alpha$ is non-singular. We set

$$
\begin{aligned}
& \Lambda_{k+1}=\Lambda_{k}+i \alpha^{-1} \Lambda_{k} j \quad\left(k \in \mathbb{N}_{0}\right) \\
& S_{k+1}=S_{k}+\alpha^{-1} S_{k} \alpha^{-*}+\alpha^{-1} \Lambda_{k} j \Lambda_{k}^{*} \alpha^{-*} \quad\left(k \in \mathbb{N}_{0}\right),
\end{aligned}
$$

where $\alpha^{-*}$ stands for $\left(\alpha^{-1}\right)^{*}$. From (3.3) and $\Lambda_{0}=\left[\begin{array}{ll}\vartheta_{1} & \vartheta_{2}\end{array}\right]$ it follows that

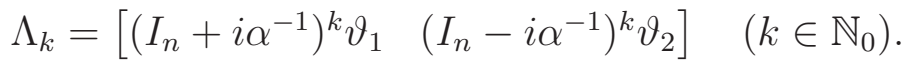


By definition $\Sigma_{k}$ is the quadruple given by

$$
\Sigma_{k}=\left\{\alpha, S_{k},\left(I_{n}+i \alpha^{-1}\right)^{k} \vartheta_{1},\left(I_{n}-i \alpha^{-1}\right)^{k} \vartheta_{2}\right\} .
$$

The next lemma shows that this quadruple is again admissible if, in addition, the pair $\left\{\alpha, \vartheta_{1}\right\}$ is controllable.

Lemma 3.2. Let $\Sigma_{0}=\left\{\alpha, S_{0}, \vartheta_{1}, \vartheta_{2}\right\}$ be an admissible quadruple. Assume that the pair $\left\{\alpha, \vartheta_{1}\right\}$ is controllable. Then $\alpha$ is non-singular, and the quadruple $\Sigma_{k}$ defined by (3.6) is admissible for each $k \in \mathbb{N}_{0}$. Moreover the pair $\left\{\alpha,\left(I_{n}+i \alpha^{-1}\right)^{k} \vartheta_{1}\right\}$ is controllable.

Proof. The fact that the pair $\left\{\alpha, \vartheta_{1}\right\}$ is controllable implies that the pair $\left\{\alpha, \Lambda_{0}\right\}$ is also controllable. But then item (ii) in Lemma A.1 tells us that $\sigma(\alpha) \subset \mathbb{C}_{+}$. In particular, $\alpha$ is non-singular, and thus the quadruple $\Sigma_{k}$ is well-defined.

Next we use (3.3) and (3.4) to prove that

$$
\alpha S_{k}-S_{k} \alpha^{*}=i \Lambda_{k} \Lambda_{k}^{*} \quad\left(k \in \mathbb{N}_{0}\right) .
$$

This will be done by induction. Since $\Lambda_{0}=\left[\begin{array}{ll}\vartheta_{1} & \vartheta_{2}\end{array}\right]$ and the quadruple $\Sigma_{0}=\left\{\alpha, S_{0}, \vartheta_{1}, \vartheta_{2}\right\}$ is admissible, the identity (3.7) holds for $k=0$. Suppose now that (3.7) holds for $k=r$. Then, using the expression for $S_{r+1}$ from (3.4), we obtain

$$
\begin{aligned}
\alpha S_{r+1}-S_{r+1} \alpha^{*}=i \Lambda_{r} \Lambda_{r}^{*} & +i \alpha^{-1} \Lambda_{r} \Lambda_{r}^{*} \alpha^{-*}+ \\
& +\Lambda_{r} j \Lambda_{r}^{*} \alpha^{-*}-\alpha^{-1} \Lambda_{r} j \Lambda_{r}^{*} .
\end{aligned}
$$

On the other hand, using (3.3) for $k=r$, we obtain

$$
\begin{aligned}
i \Lambda_{r+1} \Lambda_{r+1}^{*} & =i\left(\Lambda_{r}+i \alpha^{-1} \Lambda_{r} j\right)\left(\Lambda_{r}^{*}-i j \Lambda_{r}^{*} \alpha^{-*}\right) \\
& =i \Lambda_{r} \Lambda_{r}^{*}-\alpha^{-1} \Lambda_{r} j \Lambda_{r}^{*}+\Lambda_{r} j \Lambda_{r}^{*} \alpha^{-*}+i \alpha^{-1} \Lambda_{r} \Lambda_{r}^{*} \alpha^{-*} .
\end{aligned}
$$

Together (3.8) and (3.9) yield (3.7) for $k=r+1$, and thus (3.7) holds for all $k \in \mathbb{N}_{0}$.

Next we show that $S_{k}$ is positive definite for each $k \in \mathbb{N}_{0}$. To do this we first use that $\sigma(\alpha) \subset \mathbb{C}_{+}$. The latter inclusion and the identities (3.7) imply (cf., [26] or Theorem I.4.1 in [21]; also (A.7)) that

$$
S_{k}=\frac{1}{2 \pi} \int_{-\infty}^{\infty}\left(\lambda I_{n}-\alpha\right)^{-1} \Lambda_{k} \Lambda_{k}^{*}\left(\lambda I_{n}-\alpha^{*}\right)^{-1} d \lambda, \quad k \in \mathbb{N}_{0}
$$


Now, the inequality $S_{k}>0$ is proved by contradiction. Assuming that $S_{k} \ngtr 0$, we derive (from (3.10)) the existence of a vector $g \neq 0$ such that $g\left(\lambda I_{n}-\right.$ $\alpha)^{-1} \Lambda_{k} \equiv 0$. In particular, using the identitiy (3.5), we see that $\widetilde{g}\left(\lambda I_{n}-\right.$ $\alpha)^{-1} \vartheta_{1} \equiv 0$, where $\widetilde{g}=g\left(I_{n}+i \alpha^{-1}\right)^{k}$. The fact that $\sigma(\alpha) \subset \mathbb{C}_{+}$implies that $-i$ is not an eigenvalue of $\alpha$, and hence the matrix $I_{n}+i \alpha^{-1}$ is non-singular. But then $\widetilde{g} \neq 0$ because $g \neq 0$. On the other hand, from the Taylor expansion of $\widetilde{g}\left(\lambda I_{n}-\alpha\right)^{-1} \vartheta_{1}$ we obtain that

$$
\widetilde{g} \alpha^{p} \vartheta_{1}=0 \quad(\widetilde{g} \neq 0), \quad \text { for all } p \geq 0,
$$

which contradicts the assumption that the pair $\left\{\alpha, \vartheta_{1}\right\}$ is controllable. Thus $S_{k}$ is positive definite, and hence $\Sigma_{k}$ is admissible.

It remains to prove the pair $\left\{\alpha,\left(I_{n}+i \alpha^{-1}\right)^{k} \vartheta_{1}\right\}$ is controllable. To do this note that

$$
\operatorname{Span} \bigcup_{\nu=0}^{n-1} \operatorname{Im} \alpha^{\nu}\left(I_{n}+i \alpha^{-1}\right)^{k} \vartheta_{1}=\left(I_{n}+i \alpha^{-1}\right)^{k}\left(\operatorname{Span} \bigcup_{\nu=0}^{n-1} \operatorname{Im} \alpha^{\nu} \vartheta_{1}\right)
$$

Since the pair $\left\{\alpha, \vartheta_{1}\right\}$ is controllable, the space $\operatorname{Span} \bigcup_{\nu=0}^{n-1} \operatorname{Im} \alpha^{\nu} \vartheta_{1}$ is equal to $\mathbb{C}^{n}$. It follows that the space in the right-hand side of $(3.11)$ is also equal to $\mathbb{C}^{n}$. But then the same holds true for the space in the left-hand side of (3.11), which implies that $\left\{\alpha,\left(I_{n}+i \alpha^{-1}\right)^{k} \vartheta_{1}\right\}$ is controllable.

Note that the conditions in Lemma 3.2 imply that the matrix $S_{k}$ is nonsingular for each $k$. This allows us to define the following sequence of matrices:

$$
C_{k}=j+\Lambda_{k}^{*} S_{k}^{-1} \Lambda_{k}-\Lambda_{k+1}^{*} S_{k+1}^{-1} \Lambda_{k+1}, \quad k=0,1,2, \ldots
$$

Definition 3.3. We call the quadruple $\Sigma_{0}=\left\{\alpha, S_{0}, \vartheta_{1}, \vartheta_{2}\right\}$ strongly admissible if it is admissible and the pair $\left\{a, \vartheta_{1}\right\}$ is controllable. In this case we refer to the sequence of matrices $\left\{C_{k}\right\}_{k \in \mathbb{N}_{0}}$ in (3.12) as the pseudo-exponential potential generated by $\Sigma_{0}$.

Later we shall see (Proposition 3.6 below) that the second part of (3.1) is fulfilled for any pseudo-exponential potential $\left\{C_{k}\right\}_{k \in \mathbb{N}_{0}}$.

Remark 3.4. We note that for any continuous pseudo-exponential potential $v$ (see its definition at the beginning of Section 2) there is a strongly admissible 
quadruple which generates it. Indeed, recall that in view of Proposition 2.3 and Theorem 2.6 there is a unique solution of the inverse problem considered in Theorem 2.7. Thus, starting from the Weyl function of a system with the pseudo-exponential $v$ we recover some admissible quadruple generating this $v$ via formula (2.22) from Theorem 2.7. It is immediate from (2.22) that the corresponding pair $\left\{a, \vartheta_{1}\right\}$ is controllable, and so the recovered quadruple is strongly admissible.

Next, we present a discrete analog of formula (2.9) for the fundamental solution. See the next theorem which is a minor generalization of Theorem 0.1 in [27].

Theorem 3.5. Let $\Sigma_{0}=\left\{\alpha, S_{0}, \vartheta_{1}, \vartheta_{2}\right\}$ be a strongly admissible quadruple, and let $\left\{C_{k}\right\}_{k \in \mathbb{N}_{0}}$ be the pseudo-exponential potential generated by $\Sigma_{0}$. Then the fundamental solution $w_{k}(z)\left(k \in \mathbb{N}_{0}\right)$ of the normalized initial value problem

$$
w_{k+1}(z)=\left(I_{m}+\frac{i}{z} C_{k}\right) w_{k}(z), \quad w_{0}(z) \equiv I_{m}
$$

can be represented in the form

$$
w_{k}(z)=W_{\Sigma_{k}}(-z)\left(I_{m}+\frac{i}{z} j\right)^{k} W_{\Sigma_{0}}(-z)^{-1}
$$

where $\Sigma_{k}$ is the admissible quadruple defined by (3.6) and $W_{\Sigma_{k}}$ is the transfer function associated with $\Sigma_{k}$, that is,

$$
W_{\Sigma_{k}}(\lambda)=I_{m}+i \Lambda_{k}^{*} S_{k}^{-1}\left(\lambda I_{n}-\alpha\right)^{-1} \Lambda_{k}
$$

See the first paragraph of Section A for the definition of the transfer function associated with an admissible quadruple.

Proof. The proof is based on the following identity:

$$
W_{\Sigma_{k+1}}(\lambda)\left(I_{m}-\frac{i}{\lambda} j\right)=\left(I_{m}-\frac{i}{\lambda} C_{k}\right) W_{\Sigma_{k}}(\lambda) .
$$

This identity is the analogue of formula (2.2) in [27], and its proof follows the same line of reasoning as the proof of formula (2.2) in [27]. We omit the details. 
Next, using (3.16), we complete the proof by induction. First notice that the equality (3.14) holds for $k=0$. Next, assume (3.14) is proved for $k=r$. Then, using (3.13), (3.14) with $k=r$, and (3.16) with $k=r$ and $\lambda=-z$, we see that

$$
\begin{aligned}
w_{r+1} & =\left(I_{m}+\frac{i}{z} C_{r}\right) w_{r}(z) \\
& =\left(I_{m}+\frac{i}{z} C_{r}\right) W_{\Sigma_{r}}(-z)\left(I_{m}+\frac{i}{z} j\right)^{r} W_{\Sigma_{0}}(-z)^{-1} \\
& =W_{\Sigma_{r+1}}(-z)\left(I_{m}+\frac{i}{z} j\right)\left(I_{m}+\frac{i}{z} j\right)^{r} W_{\Sigma_{0}}(-z)^{-1} \\
& =W_{\Sigma_{r+1}}(-z)\left(I_{m}+\frac{i}{z} j\right)^{r+1} W_{\Sigma_{0}}(-z)^{-1} .
\end{aligned}
$$

Thus (3.14) holds for $k=r+1$, and so it holds for all $k \in \mathbb{N}_{0}$.

Our next proposition shows that the second equality in (3.1) is valid.

Proposition 3.6. Let $\Sigma_{0}=\left\{\alpha, S_{0}, \vartheta_{1}, \vartheta_{2}\right\}$ be a strongly admissible quadruple, and let $\left\{C_{k}\right\}_{k \in \mathbb{N}_{0}}$ be the pseudo-exponential potential generated by $\Sigma_{0}$. Then we have

$$
C_{k}=U_{k}^{*} j U_{k}, \quad \text { where } \quad U_{k}^{*} U_{k}=I_{m} \quad\left(k \in \mathbb{N}_{0}\right) .
$$

Proof. According to Lemma 3.2, $\Sigma_{k}$ is an admissible quadruple, and so we may substitute $\Sigma_{k}$ (instead of $\Sigma$ ) into formula (A.8) from Lemma A.1. Then (A.8) implies that

$$
W_{\Sigma_{k}}(\lambda) W_{\Sigma_{k}}(\bar{\lambda})^{*}=I_{m} \quad\left(k \in \mathbb{N}_{0}\right),
$$

where $\bar{\lambda}$ stands for complex conjugate for $\lambda$. Note that (3.18) makes sense only when $\lambda$ and $\bar{\lambda}$ are no poles of $W_{\Sigma_{k}}$.

It is immediate from (3.12) that $C_{k}=C_{k}^{*}$. From (3.16) and (3.18) we see that

$$
\left(I_{m}-i \lambda^{-1} C_{k}\right)\left(I_{m}+i \lambda^{-1} C_{k}\right)=\lambda^{-2}\left(\lambda^{2}+1\right) I_{m}, \quad \lambda \in \mathbb{R},
$$

that is, $C_{k}^{2}=I_{m}$. Thus, the equality $C_{k}=C_{k}^{-1}$ is valid, and we obtain the representation

$$
C_{k}=\widetilde{U}_{k} j_{k} \widetilde{U}_{k}^{*}
$$


where $\widetilde{U}_{k}$ are unitary matrices and $j_{k}$ are diagonal matrices, the entries of which take the values \pm 1 .

Next we show that we may choose $j_{k}=j$. From Lemma A.1 we know that that $\sigma(\alpha) \subset \overline{\mathbb{C}_{+}}$. In particular, $-i$ does not belong to $\sigma(\alpha)$. We first deal with the case when the same holds true for $i$. In that case (3.18) makes sense for both $\lambda=i$ and $\lambda=-i$. Let us partition the transfer matrix function $W_{\Sigma_{k}}(\lambda)$ into the two blocks, the first consisting of the first $m_{1}$ columns and the second of the remaining $m_{2}$ columns:

$$
W_{\Sigma_{k}}(\lambda)=\left[\begin{array}{ll}
\left(W_{\Sigma_{k}}(\lambda)\right)_{1} & \left(W_{\Sigma_{k}}(\lambda)\right)_{2}
\end{array}\right]
$$

Now, take $\lambda=-i$ in (3.16) and multiply the resulting identity from the right by $W_{\Sigma_{k}}(i)^{*}$. Then, using (3.18) with $\lambda=-i$, we obtain

$$
2\left(W_{\Sigma_{k+1}}(-i)\right)_{1}\left(W_{\Sigma_{k}}(i)\right)_{1}^{*}=I_{m}+C_{k} .
$$

Repeating the argument with $\lambda=i$ in place of $\lambda=-i$ we see that

$$
2\left(W_{\Sigma_{k+1}}(i)\right)_{2}\left(W_{\Sigma_{k}}(-i)\right)_{2}^{*}=I_{m}-C_{k} .
$$

Formulas (3.20) and (3.21) imply that

$$
\operatorname{rank}\left(I_{m}+C_{k}\right) \leq m_{1}, \quad \operatorname{rank}\left(I_{m}-C_{k}\right) \leq m_{2} .
$$

The existence of the representation (3.17) now easily follows from (3.19) and (3.22). Thus the proposition is proved when $i \notin \sigma(\alpha)$.

It remains to consider the case when $i$ belongs to $\sigma(\alpha)$. In this case we approximate the original quadruple $\Sigma_{0}=\left\{\alpha, S_{0}, \vartheta_{1}, \vartheta_{2}\right\}$ by a set of new quadruples $\Sigma(\varepsilon)=\left\{\alpha+\varepsilon I_{n}, S_{0}, \vartheta_{1}, \vartheta_{2}\right\}$, with $\varepsilon=\bar{\varepsilon}>0$ and $\varepsilon$ sufficiently small. These new quadruples are again strongly admissible. Thus they satisfy the conditions of Theorem 3.5 and the additional condition

$$
i \notin \sigma\left(\alpha+\varepsilon I_{n}\right)
$$

Therefore, applying the result of the previous paragraph, (3.17) is valid also in this case. Taking limits for $\varepsilon \downarrow 0$ one obtains (3.17) for the original quadruple $\Sigma_{0}$. 
Remark 3.7. It is not difficult to see that Theorem 3.5, the identities (3.7) and the representations in (3.17) hold under weaker conditions. Indeed, assume that $\alpha$ is non-singular, that $S_{0}=S_{0}^{*}$, that (3.7) holds for $k=0$, and that $\Lambda_{k}$ and $S_{k}$ are given by (3.3) and (3.4), respectively. Then the identities (3.7) are valid for $k \in \mathbb{N}_{0}$. Assuming additionally that $\operatorname{det} S_{r} \neq 0$ for $0 \leq r \leq N$, one can show that the fundamental solution $w_{k}(z)(0 \leq k \leq N)$ of the discrete system (3.13) can be represented in the form (3.14) and that (3.17) is also valid for $0 \leq k \leq N-1$.

Weyl function: the direct problem. The following result is a discrete analogue of Theorem 2.5. The special case $m_{1}=m_{2}$ of this result is a somewhat stronger version of Theorem 0.4 in [27].

Theorem 3.8. Let $\Sigma_{0}=\left\{\alpha, S_{0}, \vartheta_{1}, \vartheta_{2}\right\}$ be a strongly admissible quadruple, and let $\left\{C_{k}\right\}_{k \in \mathbb{N}_{0}}$ be the pseudo-exponential sequence generated by $\Sigma_{0}$. Then the discrete skew-selfadjoint Dirac system (3.1) has a unique Weyl function $\varphi$ which is given by the formula

$$
\varphi(z)=-i \vartheta_{1}^{*} S_{0}^{-1}\left(z I_{n}+\beta\right)^{-1} \vartheta_{2}, \quad \beta:=\alpha-i \vartheta_{2} \vartheta_{2}^{*} S_{0}^{-1} .
$$

Moreover, $\varphi$ satisfies (3.2) in the half-plane $\Im z>\frac{1}{2}$ (a finite number of points excluded).

Proof. PART 1. In order to show that $\varphi$ is a Weyl function one first compares (3.23) with (A.4) to see that $\varphi$ is the (second) function associated with $\Sigma_{0}$. Thus, the second part in (A.11) implies that

$$
\varphi(z)=b(-z) d(-z)^{-1}
$$

where $b$ and $d$ are the blocks of $W_{\Sigma_{0}}$ (see (A.10)). Using (3.24) we have

$$
W_{\Sigma_{0}}(-z)^{-1}\left[\begin{array}{c}
\varphi(z) \\
I_{m_{2}}
\end{array}\right]=W_{\Sigma_{0}}(-z)^{-1}\left[\begin{array}{c}
b(-z) \\
d(-z)
\end{array}\right] d(-z)^{-1}=\left[\begin{array}{c}
0 \\
I_{m_{2}}
\end{array}\right] d(-z)^{-1} .
$$

Next, for $k \in \mathbb{N}_{0}$, let $W_{\Sigma_{k}}$ be the transfer function of the quadruple $\Sigma_{k}$ defined by (3.6). Recall that the normalized fundamental solution $w_{k}$ of (3.1) is given 
by (3.14). Using (3.25) this yields

$$
\begin{aligned}
w_{k}(z)\left[\begin{array}{c}
\varphi(z) \\
I_{m_{2}}
\end{array}\right] & =W_{\Sigma_{k}}(-z)\left(I_{m}+\frac{i}{z} j\right)^{k} W_{\Sigma_{0}}(-z)^{-1}\left[\begin{array}{c}
\varphi(z) \\
I_{m_{2}}
\end{array}\right] \\
& =W_{\Sigma_{k}}(-z)\left(I_{m}+\frac{i}{z} j\right)^{k}\left[\begin{array}{c}
0 \\
I_{m_{2}}
\end{array}\right] d(-z)^{-1} \\
& =\left(\frac{z-i}{z}\right)^{k} W_{\Sigma_{k}}(-z)\left[\begin{array}{c}
0 \\
I_{m_{2}}
\end{array}\right] d(-z)^{-1}
\end{aligned}
$$

Since $S_{k}>0$ and $\sigma(\alpha) \subset \overline{\mathbb{C}_{+}}$(according to item (i) in Lemma 3.2), the identity (A.9) implies that

$$
W_{\Sigma_{k}}(-z)^{*} W_{\Sigma_{k}}(-z) \leq I_{m} \quad\left(z \in \mathbb{C}_{+}\right) .
$$

From (3.26) and (3.27) we easily derive (3.2) for all $z \in \mathbb{C}_{1 / 2}$, a finite number of eigenvalues excluded.

PART 2. It remains to prove the uniqueness of the Weyl function. We shall again need the identity (A.9) (for the quadruple $\Sigma_{k}$ ):

$$
W_{\Sigma_{k}}(\lambda)^{*} W_{\Sigma_{k}}(\lambda)=I_{m}-i(\lambda-\bar{\lambda}) \Lambda_{k}^{*}\left(\bar{\lambda} I_{n}-\alpha^{*}\right)^{-1} S_{k}^{-1}\left(\lambda I_{n}-\alpha\right)^{-1} \Lambda_{k} .
$$

Since the left upper block of (3.28) is nonnegative, taking into account (3.5), we obtain for $z=(-\lambda) \in \mathbb{C}_{+}$the inequality

$$
\vartheta_{1}^{*}\left(\bar{z} I_{n}+\alpha^{*}\right)^{-1}\left(I_{n}-i\left(\alpha^{*}\right)^{-1}\right)^{k} S_{k}^{-1}\left(I_{n}+i \alpha^{-1}\right)^{k}\left(z I_{n}+\alpha\right)^{-1} \vartheta_{1} \leq \frac{i}{z-\bar{z}} I_{m_{1}} .
$$

Using the same arguments as at the end of the proof of Lemma 3.2, we see that the controllability of the pair $\left\{\alpha, \vartheta_{1}\right\}$ implies the equality

$$
\operatorname{Span}_{z \in O_{\varepsilon}\left(z_{0}\right)}\left(z I_{n}+\alpha\right)^{-1} \vartheta_{1}=\mathbb{C}^{n}
$$

for any $\varepsilon$-neighborhood $O_{\varepsilon}\left(z_{0}\right)$ of any $z_{0} \in \mathbb{C}$. From (3.29) and (3.30) we see that for all $k \in \mathbb{N}_{0}$ the positive definite matrix $\left(I_{n}-i\left(\alpha^{*}\right)^{-1}\right)^{k} S_{k}^{-1}\left(I_{n}+i \alpha^{-1}\right)^{k}$ is uniformly bounded. Hence, the matrix function

$$
i(\bar{z}-z) \vartheta_{1}^{*}\left(\bar{z} I_{n}+\alpha^{*}\right)^{-1}\left(I_{n}-i\left(\alpha^{*}\right)^{-1}\right)^{k} S_{k}^{-1}\left(I_{n}+i \alpha^{-1}\right)^{k}\left(z I_{n}+\alpha\right)^{-1} \vartheta_{1}
$$


is sufficiently small for all $z \in \mathbb{C}_{M}$ and sufficiently large values of $M$. Recall that the matrix function above coincides with the left upper block of the second term on the right hand side of (3.28) (for $\lambda=-z$ ). Therefore, relations (3.14) and (3.28) yield the inequality

$$
\left[\begin{array}{ll}
I_{m_{1}} & 0
\end{array}\right] W_{\Sigma_{0}}(-z)^{*} w_{k}(z)^{*} w_{k}(z) W_{\Sigma_{0}}(-z)\left[\begin{array}{c}
I_{m_{1}} \\
0
\end{array}\right] \geq \frac{1}{2} I_{m_{1}}
$$

for $z \in \mathbb{C}_{M}$ and sufficiently large values of $M$. Thus, for sufficiently large $M$ and all $z \in \mathbb{C}_{M}$, we have

$$
\operatorname{dim} L \leq m_{2}, \quad L:=\left\{g \in \mathbb{C}^{m} \mid \sum_{k=0}^{\infty} g^{*} w_{k}(z)^{*} w_{k}(z) g<\infty\right\} .
$$

Assume now that for some $z \in \mathbb{C}_{M}$ there is a value $\widetilde{\varphi}(z)$ such that

$$
\sum_{k=0}^{\infty}\left[\widetilde{\varphi}(z)^{*} \quad I_{m_{2}}\right] w_{k}(z)^{*} w_{k}(z)\left[\begin{array}{c}
\widetilde{\varphi}(z) \\
I_{m_{2}}
\end{array}\right]<\infty, \quad \widetilde{\varphi}(z) \neq \varphi(z) .
$$

Then (3.2) and (3.33) contradict the inequality (3.32). Hence, there is no such $\widetilde{\varphi}$ and the Weyl function is unique.

Weyl function: the inverse problem. From Theorem 3.8 we know that the Weyl function of a discrete skew-selfadjoint Dirac system (3.1) with a pseudo-exponential potential generated by a strongly admissible quadruple is a strictly proper rational matrix function. The next theorem shows that the converse is also true.

Theorem 3.9. Let $\varphi$ be a strictly proper rational $m_{1} \times m_{2}$ matrix function. Then $\varphi$ is the Weyl function of a discrete skew-selfadjoint Dirac system (3.1) with a pseudo-exponential potential generated by a strongly admissible quadruple $\Sigma_{0}$. A corresponding $\Sigma_{0}=\left\{\alpha, S_{0}, \vartheta_{1}, \vartheta_{2}\right\}$ can be obtained explicitly by using the following procedure. First, we construct a minimal realization of $\varphi$ :

$$
\varphi(z)=-i \theta_{1}^{*}\left(z I_{n}+\gamma\right)^{-1} \theta_{2} .
$$

Next, we choose $X$ to be the unique positive definite solution of the Riccati equation $\gamma X-X \gamma^{*}-i X \theta_{1} \theta_{1}^{*} X+i \theta_{2} \theta_{2}^{*}=0$. Finally, we put

$$
S_{0}=I_{n}, \quad \vartheta_{1}=X^{1 / 2} \theta_{1}, \quad \vartheta_{2}=X^{-1 / 2} \theta_{2}, \quad \alpha=X^{-1 / 2} \gamma X^{1 / 2}+i \vartheta_{2} \vartheta_{2}^{*} .
$$


Proof. Given a strictly proper rational matrix function $\varphi$, Corollary A.4 shows that the procedure to recover $\Sigma_{0}=\left\{\alpha, S_{0}, \vartheta_{1}, \vartheta_{2}\right\}$ is well-defined and $\Sigma_{0}$ is strongly admissible. Moreover, according to Corollary A.4, $\varphi$ admits representation (3.23) where the quadruple $\left\{\alpha, S_{0}, \vartheta_{1}, \vartheta_{2}\right\}$ is given by (3.35). Then we know from Theorem 3.8 that $\varphi$ is the Weyl function of system (3.1) with the potential $\left\{C_{k}\right\}$ generated by $\left\{\alpha, S_{0}, \vartheta_{1}, \vartheta_{2}\right\}$.

We conclude this section with some auxiliary results on the $m \times m$ matrices $\mathcal{H}_{k}^{+}$and $\mathcal{H}_{k}^{-}$:

$$
\begin{array}{ll}
\mathcal{H}_{k}^{+}:=2 W_{\Sigma_{k}}(i) P_{1} W_{\Sigma_{k}}(-i)^{*}, & P_{1}=\left(I_{m}+j\right) / 2 ; \\
\mathcal{H}_{k}^{-}:=2 W_{\Sigma_{k}}(-i) P_{2} W_{\Sigma_{k}}(i)^{*}, & P_{2}=\left(I_{m}-j\right) / 2,
\end{array}
$$

which will be essential in the next section. Here $W_{\Sigma_{k}}$ is the transfer function of the form (3.15).

Lemma 3.10. Let $\Sigma_{0}=\left\{\alpha, S_{0}, \vartheta_{1}, \vartheta_{2}\right\}$ be a strongly admissible quadruple, let $\Sigma_{k}$ be defined by (3.6), assume that $i \notin \sigma(\alpha)$, and let $\left\{C_{k}\right\}_{k \in \mathbb{N}_{0}}$ be the pseudo-exponential potential generated by $\Sigma_{0}$. Then

$$
\begin{aligned}
& \left(I_{m}+C_{k}\right) \mathcal{H}_{k}^{-}=\mathcal{H}_{k+1}^{-}\left(I_{m}+C_{k}\right)=0, \\
& \left(I_{m}-C_{k}\right) \mathcal{H}_{k}^{+}=\mathcal{H}_{k+1}^{+}\left(I_{m}-C_{k}\right)=0 .
\end{aligned}
$$

Proof. Since $\Sigma_{0}=\left\{\alpha, S_{0}, \vartheta_{1}, \vartheta_{2}\right\}$ is admissible, we know (see Lemma A.1) that $-i \notin \sigma(\alpha)$. By assumption $i \notin \sigma(\alpha)$. This allows us to apply (3.18), first with $\lambda=i$ and next with $\lambda=-1$. It follows that both $W_{\Sigma_{k}}(i)$ and $W_{\Sigma_{k}}(-i)$ are well-defined and invertible. Moreover, we have

$$
W_{\Sigma_{k}}(i)^{-1}=W_{\Sigma_{k}}(-i)^{*} \quad \text { and } \quad W_{\Sigma_{k}}(-i)^{-1}=W_{\Sigma_{k}}(i)^{*} .
$$

Using these identities the formulas for $\mathcal{H}_{k}^{+}$and $\mathcal{H}_{k}^{-}$can be rewritten as:

$$
\mathcal{H}_{k}^{+}=2 W_{\Sigma_{k}}(i) P_{1} W_{\Sigma_{k}}(i)^{-1}, \quad \mathcal{H}_{k}^{-}=2 W_{\Sigma_{k}}(-i) P_{2} W_{\Sigma_{k}}(-i)^{-1} .
$$

Next, applying (3.16), first with $\lambda=i$ and next with $\lambda=-i$, we see that

$$
\begin{aligned}
& \left(I_{m}-C_{k}\right) W_{\Sigma_{k}}(i)=2 W_{\Sigma_{k+1}}(i) P_{2}, \\
& \left(I_{m}+C_{k}\right) W_{\Sigma_{k}}(-i)=2 W_{\Sigma_{k+1}}(-i) P_{1} .
\end{aligned}
$$


It follows that

$$
\begin{aligned}
\left(I_{m}-C_{k}\right) \mathcal{H}_{k}^{+} & =2\left(I_{m}-C_{k}\right) W_{\Sigma_{k}}(i) P_{1} W_{\Sigma_{k}}(-i)^{*} \\
& =4 W_{\Sigma_{k+1}}(i) P_{2} P_{1} W_{\Sigma_{k}}(-i)^{*}=0 \\
\left(I_{m}+C_{k}\right) \mathcal{H}_{k}^{-} & =2\left(I_{m}+C_{k}\right) W_{\Sigma_{k}}(-i) P_{2} W_{\Sigma_{k}}(i)^{*} \\
& =4 W_{\Sigma_{k+1}}(-i) P_{1} P_{2} W_{\Sigma_{k}}(i)^{*}=0 .
\end{aligned}
$$

From (3.41), we obtain

$$
\begin{aligned}
& \mathcal{H}_{k}^{+} W_{\Sigma_{k}}(i)=2 W_{\Sigma_{k}}(i) P_{1} W_{\Sigma_{k}}(i)^{-1} W_{\Sigma_{k}}(i)=2 W_{\Sigma_{k}}(i) P_{1} \\
& \mathcal{H}_{k}^{-} W_{\Sigma_{k}}(-i)=2 W_{\Sigma_{k}}(-i) P_{2} W_{\Sigma_{k}}(-i)^{-1} W_{\Sigma_{k}}(-i)=2 W_{\Sigma_{k}}(-i) P_{2}
\end{aligned}
$$

In particular, we have

$$
\mathcal{H}_{k}^{+} W_{\Sigma_{k}}(i) P_{2}=0 \quad \text { and } \quad \mathcal{H}_{k}^{-} W_{\Sigma_{k}}(-i) P_{1}=0 \quad(k \geq 0)
$$

Using (3.42) and (3.43) again and taking into account (3.48), we see that

$$
\begin{aligned}
\mathcal{H}_{k+1}^{+}\left(I_{m}-C_{k}\right) W_{\Sigma_{k}}(i) & =\mathcal{H}_{k+1}^{+}\left(I_{m}-C_{k}\right) W_{\Sigma_{k}}(i)\left(P_{1}+P_{2}\right) \\
& =2 \mathcal{H}_{k+1}^{+} W_{\Sigma_{k+1}}(i) P_{2}\left(P_{1}+P_{2}\right) \\
& =2 \mathcal{H}_{k+1}^{+} W_{\Sigma_{k+1}}(i) P_{2}=0
\end{aligned}
$$

and

$$
\begin{aligned}
\mathcal{H}_{k+1}^{-}\left(I_{m}+C_{k}\right) W_{\Sigma_{k}}(-i) & =\mathcal{H}_{k+1}^{-}\left(I_{m}+C_{k}\right) W_{\Sigma_{-k}}(-i)\left(P_{1}+P_{2}\right) \\
& =2 \mathcal{H}_{k+1}^{-} W_{\Sigma_{k+1}}(-i) P_{1}\left(P_{2}+P_{1}\right) \\
& =2 \mathcal{H}_{k+1}^{-} W_{\Sigma_{k+1}}(-i) P_{1}=0 .
\end{aligned}
$$

Formulas (3.44), (3.45), (3.49) and (3.50) yield (3.38) and (3.39).

For later purposes (see the next section) we mention that under the assumptions of Lemma 3.10 the matrices $\mathcal{H}_{k}^{+}$and $\mathcal{H}_{k}^{-}$can be rewritten as

$$
\begin{aligned}
& \mathcal{H}_{k}^{+}=I_{m}+W_{\Sigma_{k}}(i) j W_{\Sigma_{k}}(-i)^{*}, \\
& \mathcal{H}_{k}^{-}=I_{m}-W_{\Sigma_{k}}(-i) j W_{\Sigma_{k}}(i)^{*} .
\end{aligned}
$$

Indeed, these identities follow from (3.36) and (3.37) by using the two indenties in (3.40). Together (3.51) and (3.52) imply that

$$
\mathcal{H}_{k}^{+}+\left(\mathcal{H}_{k}^{-}\right)^{*}=2 I_{m} .
$$


Finally, using (3.51) we see that

$$
\begin{aligned}
\mathcal{H}_{k}^{+}=I_{m}+\left(I_{m}\right. & \left.+i \Lambda_{k}^{*} S_{k}^{-1}\left(i I_{n}-\alpha\right)^{-1} \Lambda_{k}\right) j \\
& \times\left(I_{m}+i \Lambda_{k}^{*} S_{k}^{-1}\left(-i I_{n}-\alpha\right)^{-1} \Lambda_{k}\right)^{*} \\
=I_{m}+( & \left.+i \Lambda_{k}^{*} S_{k}^{-1}\left(i I_{n}-\alpha\right)^{-1} \Lambda_{k} j\right) \\
& \times\left(I_{m}-i \Lambda_{k}^{*}\left(i I_{n}-\alpha^{*}\right)^{-1} S_{k}^{-1} \Lambda_{k}\right) \\
=I_{m}+j+ & i \Lambda_{k}^{*} S_{k}^{-1}\left(i I_{n}-\alpha\right)^{-1} \Lambda_{k} j \\
& -i j \Lambda_{k}^{*}\left(i I_{n}-\alpha^{*}\right)^{-1} S_{k}^{-1} \Lambda_{k} \\
& +\Lambda_{k}^{*} S_{k}^{-1}\left(i I_{n}-\alpha\right)^{-1} \Lambda_{k} j \Lambda_{k}^{*}\left(i I_{n}-\alpha^{*}\right)^{-1} S_{k}^{-1} \Lambda_{k} .
\end{aligned}
$$

Analogously, using (3.52), we have

$$
\begin{aligned}
\mathcal{H}_{k}^{-}=I_{m}-\left(I_{m}\right. & \left.+i \Lambda_{k}^{*} S_{k}^{-1}\left(-i I_{n}-\alpha\right)^{-1} \Lambda_{k}\right) j \\
& \times\left(I_{m}+i \Lambda_{k}^{*} S_{k}^{-1}\left(i I_{n}-\alpha\right)^{-1} \Lambda_{k}\right)^{*} \\
=I_{m}-( & \left.+i \Lambda_{k}^{*} S_{k}^{-1}\left(-i I_{n}-\alpha\right)^{-1} \Lambda_{k} j\right) \\
& \times\left(I_{m}-i \Lambda_{k}^{*}\left(-i I_{n}-\alpha^{*}\right)^{-1} S_{k}^{-1} \Lambda_{k}\right) \\
=I_{m}-j+ & i \Lambda_{k}^{*} S_{k}^{-1}\left(i I_{n}+\alpha\right)^{-1} \Lambda_{k} j \\
& -i j \Lambda_{k}^{*}\left(i I_{n}+\alpha^{*}\right)^{-1} S_{k}^{-1} \Lambda_{k} \\
& -\Lambda_{k}^{*} S_{k}^{-1}\left(i I_{n}+\alpha\right)^{-1} \Lambda_{k} j \Lambda_{k}^{*}\left(i I_{n}+\alpha^{*}\right)^{-1} S_{k}^{-1} \Lambda_{k} .
\end{aligned}
$$

\section{Generalized discrete Heisenberg magnet model and its auxiliary linear systems}

This section is devoted to the generalized discrete Heisenberg magnet model. We apply the quadruples considered in the previous section in order to construct explicit solutions of this model. Given potential $\left\{C_{k}(t)\right\}$ from such solution and using the results from the previous section, we also express explicitly evolution of the Weyl function of the auxiliary linear system, which coincides with system (3.1).

\subsection{Generalized discrete Heisenberg magnet model}

For the case that $m_{1}=m_{2}=1$, system (3.1) is an auxiliary linear system for the integrable isotropic Heisenberg magnet model [47] (see also [14, Part 
II, Section 1.2]). More precisely, it was shown in [14,47] that the isotropic Heisenberg magnet model is equivalent to the compatibility condition

$$
\frac{d}{d t} G_{k}=F_{k+1} G_{k}-G_{k} F_{k}
$$

of the auxiliary systems

$$
\begin{aligned}
y_{k+1} & =G_{k} y_{k}, \quad G_{k}(t, z):=I_{m}+\frac{i}{z} C_{k}(t) \\
\frac{d}{d t} y_{k} & =F_{k} y_{k}, \quad F_{k}(t, z):=-\frac{H_{k}^{+}(t)}{z+i}-\frac{H_{k}^{-}(t)}{z-i},
\end{aligned}
$$

where $m=2$,

$$
H_{k}^{ \pm}(t)=f_{k}(t)\left(I_{2} \pm C_{k}(t)\right)\left(I_{2} \pm C_{k-1}(t)\right)
$$

$f_{k}$ is a scalar function depending on $C_{k-1}$ and $C_{k}, k>0, C_{k}$ has the form

$$
C_{k}=\left[\begin{array}{cc}
c_{k}^{3} & c_{k}^{1}-i c_{k}^{2} \\
c_{k}^{1}+i c_{k}^{2} & -c_{k}^{3}
\end{array}\right], \quad\left(c_{k}^{1}\right)^{2}+\left(c_{k}^{2}\right)^{2}+\left(c_{k}^{2}\right)^{2}=1,
$$

and the numbers $c_{k}^{1}, c_{k}^{2}$ and $c_{k}^{3}$ are real valued. It is easy to see that representation (4.5) is equivalent to the conditions $C_{k}=C_{k}^{*}, \operatorname{det} C_{k}=1$ and $\operatorname{Tr} C_{k}=0$, where Tr means trace. These conditions are in turn equivalent to the conditions $C_{k}=C_{k}^{*}$ and the eigenvalues of $C_{k}$ equal 1 and -1 . Hence, (4.5) is equivalent to the second part of (3.1), where $m_{1}=m_{2}=1$ (see [27]). Moreover, the second part of (3.1) yields relations

$$
I_{2}+C_{k}=2 U_{k} P_{1} U_{k}^{*}, \quad I_{2}-C_{k}=2 U_{k} P_{2} U_{k}^{*},
$$

where $P_{1}=\left(I_{2}+j\right) / 2, P_{2}=\left(I_{2}-j\right) / 2$ and $P_{1} P_{2}=P_{2} P_{1}=0$. Therefore, (4.4) and (4.6) imply that $H_{k}^{ \pm}$has rank 1 , that

$$
\left(I_{2} \mp C_{k}(t)\right) H_{k}^{ \pm}(t)=H_{k}^{ \pm}(t)\left(I_{2} \mp C_{k-1}(t)\right)=0,
$$

and that (4.7) defines matrices $H_{k}^{+}$and $H_{k}^{-}$up to scalar factors.

Generalized Heisenberg magnet models are also actively studied (see, e.g., $[13,25,29,46,48,49])$, including the case of the continuous generalized Heisenberg magnet model, where the $m \times m$ matrix function $C$ (an analog of $C_{k}$ ) satisfies the condition $C=C^{*}=C^{-1}$ (see [13, Remark 5] and references 
therein). Using an analog of condition (4.7) instead of (4.4), we consider the following generalized discrete Heisenberg magnet model (DGHM):

$$
\begin{aligned}
i \frac{d}{d t} C_{k}(t)=\left(H_{k+1}^{-}(t)-H_{k+1}^{+}(t)\right) C_{k}(t) & \\
& -C_{k}(t)\left(H_{k}^{-}(t)-H_{k}^{+}(t)\right),
\end{aligned}
$$

where

$$
\begin{aligned}
& \left(I_{m}+C_{k}(t)\right) H_{k}^{-}(t)=H_{k+1}^{-}(t)\left(I_{m}+C_{k}(t)\right)=0 \\
& \left(I_{m}-C_{k}(t)\right) H_{k}^{+}(t)=H_{k+1}^{+}(t)\left(I_{m}-C_{k}(t)\right)=0
\end{aligned}
$$

and

$$
C_{k}(t)=U_{k}(t) j U_{k}(t)^{*}, \quad U_{k}(t) U_{k}(t)^{*}=I_{m}, \quad k \in \mathbb{N}_{0} .
$$

The next proposition shows that (4.8) coincides with (4.1).

Proposition 4.1. Under conditions (4.9)-(4.11), the discrete generalized Heisenberg magnet model (4.8) is equivalent to the compatibility condition (4.1) of the auxiliary systems (4.2) and (4.3).

Proof. In view of the definitions of $G_{k}$ and $F_{k}$, we rewrite (4.1) in the form

$$
\frac{i}{z} \frac{d}{d t} C_{k}=\left(I_{m}+\frac{i}{z} C_{k}\right)\left(\frac{H_{k}^{+}}{z+i}+\frac{H_{k}^{-}}{z-i}\right)-\left(\frac{H_{k+1}^{+}}{z+i}+\frac{H_{k+1}^{-}}{z-i}\right)\left(I_{m}+\frac{i}{z} C_{k}\right) .
$$

Using the equality $\frac{ \pm i}{z(z \pm i)}=\frac{1}{z}-\frac{1}{z \pm i}$, after simple transformations we see that (4.12) is equivalent to the relation

$$
\begin{aligned}
i \frac{d}{d t} C_{k}= & \left(H_{k+1}^{-}-H_{k+1}^{+}\right) C_{k}-C_{k}\left(H_{k}^{-}-H_{k}^{+}\right) \\
& +\frac{z}{z+i}\left(\left(I_{m}-C_{k}\right) H_{k}^{+}-H_{k+1}^{+}\left(I_{m}-C_{k}\right)\right) \\
& +\frac{z}{z-i}\left(\left(I_{m}+C_{k}\right) H_{k}^{-}-H_{k+1}^{-}\left(I_{m}+C_{k}\right)\right) .
\end{aligned}
$$

Taking into account the equalities (4.9) and (4.11), we derive that the terms in the second and third lines of (4.13) both turn to zero, and therefore (4.13) is equivalent to (4.8). 
In order to construct explicit solutions of DGHM, we introduce an additional parameter $t$ into the quadruples generating potentials $\left\{C_{k}(t)\right\}$. Namely, we consider quadruples $\Sigma_{t}=\Sigma_{t, 0}=\left\{\alpha, S_{0}(t), \vartheta_{1}(t), \vartheta_{2}(t)\right\}$. Here $\alpha$ is constant and the dependence of the quadruples on $t$ is described by the equations

$$
\begin{aligned}
\left(\frac{d}{d t} \Lambda_{0}\right)(t)=- & 2\left(\left(\alpha-i I_{n}\right)^{-1} \Lambda_{0}(t) P_{1}+\left(\alpha+i I_{n}\right)^{-1} \Lambda_{0}(t) P_{2}\right) \\
P_{1}:=\left(I_{m}+j\right) / 2, \quad P_{2}:=\left(I_{m}-j\right) / 2 & \\
\left(\frac{d}{d t} S_{0}\right)(t)=- & \left(\left(\alpha-i I_{n}\right)^{-1} S_{0}(t)+\left(\alpha+i I_{n}\right)^{-1} S_{0}(t)\right. \\
+ & S_{0}(t)\left(\alpha^{*}+i I_{n}\right)^{-1}+S_{0}(t)\left(\alpha^{*}-i I_{n}\right)^{-1} \\
+ & 2\left(\alpha^{2}+I_{n}\right)^{-1}\left(\alpha \Lambda_{0}(t) j \Lambda_{0}(t)^{*}+\Lambda_{0}(t) j \Lambda_{0}(t)^{*} \alpha^{*}\right) \\
& \left.\times\left(\left(\alpha^{*}\right)^{2}+I_{n}\right)^{-1}\right) .
\end{aligned}
$$

Formula (4.14) yields the equalities

$$
\begin{aligned}
& \Lambda_{0}(t)=\left[\begin{array}{ll}
\vartheta_{1}(t) & \vartheta_{2}(t)
\end{array}\right], \\
& \vartheta_{1}(t)=e^{-2 t\left(\alpha-i I_{n}\right)^{-1}} \vartheta_{1}(0), \quad \vartheta_{2}(t)=e^{-2 t\left(\alpha+i I_{n}\right)^{-1}} \vartheta_{2}(0) \text {. }
\end{aligned}
$$

We assume for simplicity that $\Sigma_{0,0}$ is strongly admissible and that $i \notin \sigma(\alpha)$. Then, the quadruples $\Sigma_{t, 0}$ are well-defined and uniquely determined by the initial quadruple $\Sigma_{0,0}$. Moreover, all $\Sigma_{t, 0}$ are strongly admissible (see Subsection 4.2). It was shown in Section 3 that the strongly admissible quadruples $\Sigma_{t, 0}$ determine strongly admissible quadruples $\Sigma_{t, k}$ using relations (3.3) and (3.4), and that in this way $\Sigma_{t, 0}$ generate potentials $\left\{C_{k}(t)\right\}$. Since $\Sigma_{t, 0}$ are determined by $\Sigma_{0,0}$ we also say that $\Sigma_{t, k}$ are determined by $\Sigma_{0,0}$ and that potentials $\left\{C_{k}(t)\right\}$ are generated by $\Sigma_{0,0}$. The following theorem is our main result in this section.

Theorem 4.2. Let $\Sigma_{0,0}=\left\{\alpha, S_{0}, \vartheta_{1}, \vartheta_{2}\right\}$ be a strongly admissible quadruple, and assume that $i \notin \sigma(\alpha)$. Then for all $t \in \mathbb{R}$ and $k \in \mathbb{N}_{0}$ the quadruples $\Sigma_{t, k}$ determined by $\Sigma_{0,0}$ are strongly admissible. Furthermore, the matrix functions

$$
\begin{aligned}
& H_{k}^{+}(t):=\mathcal{H}_{k}^{+}(t)=W_{\Sigma_{t, k}}(i)\left(I_{m}+j\right) W_{\Sigma_{t, k}}(-i)^{*}, \\
& H_{k}^{-}(t):=\mathcal{H}_{k}^{-}(t)=W_{\Sigma_{t, k}}(-i)\left(I_{m}-j\right) W_{\Sigma_{t, k}}(i)^{*}
\end{aligned}
$$

are well-defined and satisfy DGHM (4.8) and conditions (4.9) and (4.10). 
Recall that matrix functions $\mathcal{H}_{k}^{ \pm}$were introduced and studied in the last part of Section 3. The proof of Theorem 4.2 and some related results on discrete Dirac systems depending on an additional continuous time parameter are given in the next subsection.

Finally, we note that in view of (4.18) we have the following corollary of Theorems 3.8 and 4.2.

Corollary 4.3. Under the conditions of Theorem 4.2, the evolution of the Weyl function $\varphi(t, z)$ of the system (4.2) is given by the formula

$$
\begin{aligned}
& \varphi(t, z)=-i \vartheta_{1}^{*} e^{-2 t\left(\alpha^{*}+i I_{n}\right)^{-1}} S_{0}(t)^{-1}\left(z I_{n}+\beta(t)\right)^{-1} e^{-2 t\left(\alpha+i I_{n}\right)^{-1}} \vartheta_{2}, \\
& \beta(t):=\alpha-i e^{-2 t\left(\alpha+i I_{n}\right)^{-1}} \vartheta_{2} \vartheta_{2}^{*} e^{-2 t\left(\alpha^{*}-i I_{n}\right)^{-1}} S_{0}(t)^{-1}, \vartheta_{k}:=\vartheta_{k}(0)(k=1,2) .
\end{aligned}
$$

\subsection{Discrete Dirac systems depending on an additional continuous time parameter}

Let $\Sigma=\left\{\alpha, S_{0}, \vartheta_{1}, \vartheta_{2}\right\}$ be a strongly admissible quadruple, and assume that $i \notin \sigma(\alpha)$. We shall associate with $\Sigma$ a family of strongly admissible quadruples depending on two parameters $t$ and $k$, where $t \in \mathbb{R}_{+}$and $k \in \mathbb{N}_{0}$. This will be done in two steps.

Step 1: COnstruction of $\Sigma_{t}$. In this first step we just require $\Sigma$ to be admissible, not necessarily strongly admissible. From item (i) in Lemma A.1 we know that $-i \notin \sigma(\alpha)$. Together with the assumption that $i \notin \sigma(\alpha)$ this implies that both $\alpha-i I_{n}$ and $\alpha+i I_{n}$ are invertible. This allows us to define $\Lambda(t)=\Lambda_{0}(t)$ and $S(t)=S_{0}(t)$ using (4.18) (and (4.17)) and (4.16), respectively.

Lemma 4.4. Let $\Sigma=\left\{\alpha, S_{0}, \vartheta_{1}, \vartheta_{2}\right\}$ be an admissible quadruple, and assume that $i \notin \sigma(\alpha)$. Let $\vartheta_{j}(t), j=1,2$, and $S(t)$ be defined by (4.18) and (4.16), respectively. Then on some interval interval $-\varepsilon_{1}<t<\varepsilon_{2}$ the quadruple

$$
\Sigma_{t}=\left\{\alpha, S(t), \vartheta_{1}(t), \vartheta_{2}(t)\right\}
$$

is admissible too. Moreover, if $\Sigma$ is strongly admissible, then the same holds true for the quadruple $\Sigma_{t}$ for each $t \in \mathbb{R}$.

Proof. Since $S_{0}$ is positive definite, the same holds true for $S(t)$ provided $t$ is sufficiently small. Put

$$
\Upsilon(t):=\alpha S(t)-S(t) \alpha^{*}-i \Lambda(t) \Lambda(t)^{*} .
$$


Here $\Lambda(t)$ is defined by (4.17). Since $\left\{\alpha, S_{0}, \vartheta_{1}, \vartheta_{2}\right\}$ is an admissible quadruple, it follows that $\Upsilon(0)=0$. After some easy transformations, using (4.17) and (4.16) and differentiating the right-hand side of (4.22), we see that $\Upsilon$ satisfies the linear differential equation

$$
\begin{aligned}
\frac{d}{d t} \Upsilon(t)=-\left(\alpha+i I_{n}\right)^{-1}\left(\alpha \Upsilon(t)+\Upsilon(t) \alpha^{*}\right)\left(\alpha^{*}-i I_{n}\right)^{-1}+ \\
-\left(\alpha-i I_{n}\right)^{-1}\left(\alpha \Upsilon(t)+\Upsilon(t) \alpha^{*}\right)\left(\alpha^{*}+i I_{n}\right)^{-1}
\end{aligned}
$$

Since the initial value $\Upsilon(0)$ is zero, it follows that $\Upsilon(t) \equiv 0$, that is,

$$
\alpha S(t)-S(t) \alpha^{*}=i \Lambda(t) \Lambda(t)^{*} .
$$

Using (4.18) and (4.17), we conclude that $\left\{\alpha, S(t), \vartheta_{1}(t), \vartheta_{2}(t)\right\}$ is an admissible quadruple.

Next, assume $\Sigma$ is strongly admissible. The identity (4.24) was already proved above. By Definition 3.3, the fact that $\Sigma$ is strongly admissible means that the pair $\alpha$ and $\vartheta_{1}$ is controllable. But then the first equality in (4.18) tells us that

$$
\operatorname{Span} \bigcup_{\nu=0}^{n-1} \operatorname{Im} \alpha^{\nu} \vartheta_{1}(t)=e^{-2 t\left(\alpha-i I_{n}\right)^{-1}}\left(\operatorname{Span} \bigcup_{\nu=0}^{n-1} \operatorname{Im}\left(\alpha^{\nu} \vartheta_{1}(0)\right)=\mathbb{C}^{n},\right.
$$

that is, the pair $\alpha$ and $\vartheta_{1}(t)$ is also controllable. Furthermore, since $\Sigma$ is strongly admissible item (ii) of Lemma A.1 shows that $\sigma(\alpha) \subset \mathbb{C}_{+}$. From (4.24) and $\sigma(\alpha) \subset \mathbb{C}_{+}$follows representation (A.7) of $S_{0}(t)=S(t)$. Since the pair $\alpha$ and $\vartheta_{1}(t)$ is controllable representation (A.7) implies that $S(t)>0$. Hence, again using Definition 3.3, the quadruple $\Sigma_{t}$ is strongly admissible.

Step 2: COnstruction of $\Sigma_{t, k}$. Let $\Sigma=\left\{\alpha, S, \vartheta_{1}, \vartheta_{2}\right\}$ be a strongly admissible quadruple, and assume that $i \notin \sigma(\alpha)$. Let $\Sigma_{t}$ be the quadrupple defined by (4.21). Hence, according to the previous lemma, the quadruple $\Sigma_{t}$ is also strongly admissible for each $t \in \mathbb{R}$. But then we can apply the results of Section 3 to associate with each $\Sigma_{t}$ a family of quadruples $\Sigma_{t, k}, k \in \mathbb{N}_{0}$.

Since $\Sigma_{t}$ is strongly admissible, we know from Lemma 3.2 that $\alpha$ is invertible. In fact, by item (ii) in Lemma A.1, all eigenvalues of $\alpha$ are in $\mathbb{C}_{+}$. Following the constructions given in Section 3, put

$$
\begin{gathered}
\vartheta_{1}(t, k)=\left(I_{n}+i \alpha^{-1}\right)^{k} \vartheta_{1}(t), \quad \vartheta_{2, k}(t)=\left(I_{n}-i \alpha^{-1}\right)^{k} \vartheta_{2}(t), \\
\Lambda(t, k)=\left[\begin{array}{ll}
\vartheta_{1}(t, k) & \vartheta_{2}(t, k)
\end{array}\right] .
\end{gathered}
$$


Furthermore, define $S(t, k)$ by

$$
\begin{aligned}
& \begin{array}{l}
S(t, k+1)=S(t, k)+\alpha^{-1} S(t, k) \alpha^{-*}+ \\
\quad+\alpha^{-1} S(t, k) \alpha^{-*} \Lambda(t, k) j \Lambda(t, k)^{*}, \quad k=0,1,2, \ldots,
\end{array} \\
& S(t, 0)=S(t) .
\end{aligned}
$$

Using these matrices we set

$$
\Sigma_{t, k}=\left\{\alpha, S(t, k),\left(I_{n}+i \alpha^{-1}\right)^{k} \vartheta_{1}(t),\left(I_{n}-i \alpha^{-1}\right)^{k} \vartheta_{2}(t)\right\} .
$$

By Lemma 3.2, the quadruple $\Sigma_{t, k}$ is also strongly admissible.

Let $\Sigma=\left\{\alpha, S, \vartheta_{1}, \vartheta_{2}\right\}$ be a strongly admissible quadruple, and let $\Sigma_{t}$ be the strongly admissible quadruple defined by (4.21). Following (3.12) put

$$
\begin{aligned}
& C_{k}(t)=j+\Lambda(t, k)^{*} S(t, k)^{-1} \Lambda(t, k)+ \\
& \quad-\Lambda(t, k+1)^{*} S(t, k+1)^{-1} \Lambda(t, k+1), \quad k \in \mathbb{N}_{0} .
\end{aligned}
$$

Then, by Definition 3.3, the sequence $\left\{C_{k}(t)\right\}_{k \in \mathbb{N}_{0}}$ is the pseudo-expontential potential generated by $\Sigma_{t}$. The corresponding SkDDS is given by

$$
y_{k+1}(t, z)=\left(I_{m}+i z^{-1} C_{k}(t)\right) y_{k}(t, z) \quad\left(k \in \mathbb{N}_{0}\right) .
$$

Furthermore, we know (see Propositon 3.6) that $C_{k}(t)$ admits a factorization of the form:

$$
C_{k}(t)=U_{k}(t) j U_{k}(t)^{*}, \quad U_{k}(t) U_{k}(t)^{*}=I_{m}, \quad k \in \mathbb{N}_{0} .
$$

We also note that (4.9) and (4.10) are immediate consequences of the identities (3.38) and (3.39). Indeed, since $\Sigma_{t}=\left\{\alpha, S(t), \vartheta_{1}(t), \vartheta_{2}(t)\right\}$ is strongly admissible, we just apply Lemma 3.10 with $\Sigma_{t}=\Sigma_{t, 0}$ in place of $\Sigma_{0}$. Thus it remains to prove the identity (4.8) in order to prove Theorem 4.2. First, we formulate a proposition, which will be proved later.

Proposition 4.5. Let $\Sigma=\left\{\alpha, S_{0}, \vartheta_{1}, \vartheta_{2}\right\}$ be a strongly admissible quadruple, assume that $i \notin \sigma(\alpha)$, and let $\Sigma_{t, k}$ be the strongly admissible quadruple defined by (4.28), where $k \in \mathbb{N}_{0}$ and $t \in \mathbb{R}$. Then the function $Y_{k}(t, z)$ defined by

$$
Y_{k}(t, z)=W_{\Sigma_{t, k}}(-z)\left(I_{m}+\frac{i}{z} j\right)^{k} \exp \left\{-2 t\left(\frac{P_{1}}{z+i}+\frac{P_{2}}{z-i}\right)\right\},
$$

where $z \neq \pm i$ and $z \notin \sigma(-\alpha)$, is a solution of equation (4.2) as well as of equation (4.3). 
Proof of Theorem 4.2. As soon as Proposition 4.5 is proved we can prove Theorem 4.2 by using Proposition 4.1. Indeed, assume that the function $Y$ defined by (4.30) satisfies (4.2) and (4.3). Then

$$
\frac{d}{d t} Y_{k+1}(t, z)=F_{k+1}(t, z) Y_{k+1}(t, z)=F_{k+1}(t, z) G_{k}(t, z) Y_{k}(t, z),
$$

and

$$
\begin{aligned}
\frac{d}{d t} Y_{k+1}(t, z) & =\frac{d}{d t}\left(G_{k}(t, z) Y_{k}(t, z)\right) \\
& =\left(\frac{d}{d t} G_{k}(t, z)\right) Y_{k}(t, z)+G_{k}(t, z) \frac{d}{d t} Y_{k}(t, z) \\
& =\left(\left(\frac{d}{d t} G_{k}(t, z)\right)+G_{k}(t, z) F_{k}(t, z)\right) Y_{k}(t, z)
\end{aligned}
$$

We conclude that

$$
\left(\frac{d}{d t} G_{k}(t, z)\right) Y_{k}(t, z)=\left(F_{k+1}(t, z) G_{k}(t, z)-G_{k}(t, z) F_{k}(t, z)\right) Y_{k}(t, z) .
$$

Finally, note that $z \neq \pm i$ and $z \notin \sigma(-\alpha)$ imply that $Y_{k}(t, z)$ is non-singular. But then the above identity yields the compatibility equation (4.1), and we can apply Proposition 4.1 to get the identity (4.8).

In order to prove Proposition 4.5 we need to compute the derivatives

$$
\frac{d}{d t} \Lambda(t, k), \quad \frac{d}{d t} S(t, k), \quad \frac{d}{d t} \Lambda(t, k)^{*} S(t, k)^{-1}, \quad \frac{d}{d t} W_{\Sigma_{t, k}} .
$$

This will be done in a couple of steps.

The FIRST DERIVATIVE IN (4.31). Recall that $\Lambda(t, k)$ is given by $(4.26)$ and (4.27). It follows that

$$
\frac{d}{d t} \Lambda(t, k)=\left[\left(I_{n}+i \alpha^{-1}\right)^{k}\left(\frac{d}{d t} \vartheta_{1}(t)\right) \quad\left(I_{n}-i \alpha^{-1}\right)^{k}\left(\frac{d}{d t} \vartheta_{2}(t)\right)\right] .
$$

But then, using (4.18) and (4.17), we see that

$$
\begin{aligned}
& \frac{d}{d t} \Lambda(t, k)= \\
& \quad=-2\left[\begin{array}{ll}
\left(\alpha-i I_{n}\right)^{-1}\left(I_{n}+i \alpha^{-1}\right)^{k} \vartheta_{1}(t) & \left(\alpha+i I_{n}\right)^{-1}\left(I_{n}-i \alpha^{-1}\right)^{k} \vartheta_{2}(t)
\end{array}\right] \\
& \left.\quad=-2\left[\alpha-i I_{n}\right)^{-1} \vartheta_{1}(t, k) \quad\left(\alpha+i I_{n}\right)^{-1} \vartheta_{2}(t, k)\right] \\
& \left.\quad=-2\left(\alpha-i I_{n}\right)^{-1} \Lambda(t, k) P_{1}+\left(\alpha+i I_{n}\right)^{-1} \Lambda(t, k) P_{2}\right)
\end{aligned}
$$


Here $P_{1}$ and $P_{2}$ are the projctions defined in (4.19) and (4.20), respectively. THE SECOND DERIVATIVE IN (4.31). In order to compute the second derivative in (4.31) we present an alternative way to obtain the quadruple $\Sigma_{t, k}$ given by (4.28). As before, let $\Sigma=\left\{\alpha, S_{0}, \vartheta_{1}, \vartheta_{2}\right\}$ be a strongly admissible quadruple, and assume that $i \notin \sigma(\alpha)$. We know that $\sigma(\alpha) \subset \mathbb{C}_{+}$. In a particular $\alpha$ is non-singular. As in Section 3, let

$$
\Sigma_{k}=\left\{\alpha, S_{k},\left(I_{n}+i \alpha^{-1}\right)^{k} \vartheta_{1},\left(I_{n}-i \alpha^{-1}\right)^{k} \vartheta_{2}\right\}
$$

From Lemma 3.2 we know that $\Sigma_{k}$ is again a strongly admissible quadruple. The assumption $i \notin \sigma(\alpha)$ allows us to apply Lemma 4.4 to $\Sigma_{k}$ in place of $\Sigma$. Put

$$
\begin{aligned}
& \tilde{\vartheta}_{1}(k, t)=e^{-2 t\left(\alpha-i I_{n}\right)^{-1}}\left(I_{n}+i \alpha^{-1}\right)^{k} \vartheta_{1}, \\
& \tilde{\vartheta}_{2}(k, t)=e^{-2 t\left(\alpha+i I_{n}\right)^{-1}}\left(I_{n}-i \alpha^{-1}\right)^{k} \vartheta_{1}, \\
& \tilde{\Lambda}(k, t)=\left[\begin{array}{ll}
\tilde{\vartheta}_{1}(k, t) & \tilde{\vartheta}_{2}(k, t)
\end{array}\right],
\end{aligned}
$$

and let $\tilde{S}(k, t)$ be given by

$$
\begin{aligned}
& \frac{d}{d t} \tilde{S}(k, t)= \\
& =-\left[\left(\alpha-i I_{n}\right)^{-1} \tilde{S}(k, t)+\left(\alpha+i I_{n}\right)^{-1} \tilde{S}(k, t)+\right. \\
& +\tilde{S}(k, t)\left(\alpha^{*}+i I_{n}\right)^{-1}+\tilde{S}(k, t)\left(\alpha^{*}-i I_{n}\right)^{-1}+ \\
& \left.+2\left(\alpha^{2}+I_{n}\right)^{-1}\left(\alpha \tilde{\Lambda}(k, t) j \tilde{\Lambda}(k, t)^{*}+\tilde{\Lambda}(k, t) j \tilde{\Lambda}(k, t)^{*} \alpha^{*}\right)\left(\left(\alpha^{*}\right)^{2}+I_{n}\right)^{-1}\right] \text {, } \\
& \tilde{S}(k, 0)=S_{k} \text {. }
\end{aligned}
$$

Note that (4.36) is the analogue of (4.16) with $\Sigma_{k}$ in place of $\Sigma$. Thus, by Lemma 4.4, the quadruple

$$
\Sigma_{k, t}=\left\{\alpha, \tilde{S}(k, t), \tilde{\vartheta}_{1}(k, t), \tilde{\vartheta}_{2}(k, t)\right\}
$$

is strongly admissible. 
Lemma 4.6. For each $k \in \mathbb{N}_{0}$ and $t \in \mathbb{R}$ we have $\Sigma_{t, k}=\Sigma_{k, t}$. In particular,

$$
\begin{aligned}
& \frac{d}{d t} S(t, k)= \\
& \begin{array}{l}
=-\left[\left(\alpha-i I_{n}\right)^{-1} S(t, k)+\left(\alpha+i I_{n}\right)^{-1} S(t, k)+\right. \\
\quad+S(t, k)\left(\alpha^{*}+i I_{n}\right)^{-1}+S(t, k)\left(\alpha^{*}-i I_{n}\right)^{-1}+ \\
\left.\quad+2\left(\alpha^{2}+I_{n}\right)^{-1}\left(\alpha \Lambda(t, k) j \Lambda(t, k)^{*}+\Lambda(t, k) j \Lambda(t, k)^{*} \alpha^{*}\right)\left(\left(\alpha^{*}\right)^{2}+I_{n}\right)^{-1}\right] .
\end{array}
\end{aligned}
$$

Proof. From (4.18) and (4.26) and the identities (4.33) and (4.34) we see that

$$
\begin{aligned}
& \vartheta_{1}(t, k)=\tilde{\vartheta}_{1}(k, t)=e^{-2 t\left(\alpha-i I_{n}\right)^{-1}}\left(I_{n}+i \alpha^{-1}\right)^{k} \vartheta_{1}, \\
& \vartheta_{2}(t, k)=\tilde{\vartheta}_{2}(k, t)=e^{-2 t\left(\alpha+i I_{n}\right)^{-1}}\left(I_{n}-i \alpha^{-1}\right)^{k} \vartheta_{2} .
\end{aligned}
$$

It follows that $\Lambda(t, k)=\tilde{\Lambda}(k, t)$. Recall that $\Sigma_{t, k}$ and $\Sigma_{k, t}$ are both strongly admissible. Then item (ii) in Lemma A.1 tells us that $S(t, k$ and $\tilde{S}(k, t)$ are uniquely determined by $\Lambda(t, k)$ and $\tilde{\Lambda}(k, t)$. Thus $S(t, k$ and $\tilde{S}(k, t)$ coincide, and hence $\Sigma_{t, k}=\Sigma_{k, t}$. Thus $\Sigma_{t, k}=\Sigma_{k, t}$.

Finally, using $\Lambda(t, k)=\tilde{\Lambda}(k, t)$ and $S(t, k)=\tilde{S}(k, t)$, we see that (4.37) follows from (4.36).

The ThIRD DeRIVATIVE IN (4.31). From (4.32) and (4.37) we shall derive the identity

$$
\begin{aligned}
\frac{d}{d t}\left(\Lambda(t, k)^{*} S(t, k)^{-1}\right)=H_{k}^{+} & (t) \Lambda(t, k)^{*} S(t, k)^{-1}\left(\alpha-i I_{n}\right)^{-1}+ \\
& +H_{k}^{-}(t) \Lambda(t, k)^{*} S(t, k)^{-1}\left(\alpha+i I_{n}\right)^{-1} .
\end{aligned}
$$

Here $H_{k}^{+}$and $H_{k}^{-}$are the functions given by (4.19) and (4.20), respectively. To do this, note that according to (4.32) we have

$$
\begin{aligned}
\frac{d}{d t}\left(\Lambda(t, k)^{*} S(t, k)^{-1}\right)=- & 2 P_{1} \Lambda(t, k)^{*}\left(\alpha^{*}+i I_{n}\right)^{-1}+ \\
& -2 P_{2} \Lambda(t, k)^{*}\left(\alpha^{*}-i I_{n}\right)^{-1}+ \\
& -\Lambda(t, k)^{*} S(t, k)^{-1}\left(\frac{d}{d t} S(t, k)\right) S(t, k)^{-1} .
\end{aligned}
$$

Identity (3.7) implies that

$$
\begin{aligned}
& \left(\alpha^{*} \pm i I_{n}\right)^{-1} S(t, k)^{-1}=S(t, k)^{-1}\left(\alpha \pm i I_{n}\right)^{-1}+ \\
& \quad+i\left(\alpha^{*} \pm i I_{n}\right)^{-1} S(t, k)^{-1} \Lambda(t, k) \Lambda(t, k)^{*} S(t, k)^{-1}\left(\alpha \pm i I_{n}\right)^{-1} .
\end{aligned}
$$


By using (4.37), (4.40) and the equalities

$$
\begin{aligned}
& 2\left(\alpha^{2}+I_{n}\right)^{-1}=i\left(\left(\alpha+i I_{n}\right)^{-1}-\left(\alpha-i I_{n}\right)^{-1}\right), \\
& 2\left(\alpha^{2}+I_{n}\right)^{-1} \alpha=\left(\alpha-i I_{n}\right)^{-1}+\left(\alpha+i I_{n}\right)^{-1},
\end{aligned}
$$

after some calculations, we see that (4.39) can be rewritten in the form:

$$
\begin{aligned}
& \frac{d}{d t}\left(\Lambda(t, k)^{*} S(t, k)^{-1}\right)= \\
& \quad=V_{k}^{+}(t) \Lambda(t, k)^{*} S(t, k)^{-1}\left(\alpha-i I_{n}\right)^{-1}+V_{k}^{-}(t) \Lambda(t, k)^{*} S(t, k)^{-1}\left(\alpha+i I_{n}\right)^{-1}
\end{aligned}
$$

Here

$$
\begin{aligned}
& V_{k}^{+}(t)=I_{m}+j-i \Lambda(t, k)^{*} S(t, k)^{-1}\left(\alpha-i I_{n}\right)^{-1} \Lambda(t, k) j+ \\
& \quad+i j \Lambda(t, k)^{*}\left(\alpha^{*}-i I_{n}\right)^{-1} S(t, k)^{-1} \Lambda(t, k)+\Lambda(t, k)^{*} S(t, k)^{-1} \times \\
& \quad \times\left(\alpha-i I_{n}\right)^{-1} \Lambda(t, k) j \Lambda(t, k)^{*}\left(\alpha^{*}-i I_{n}\right)^{-1} S(t, k)^{-1} \Lambda(t, k),
\end{aligned}
$$

and

$$
\begin{aligned}
& V_{k}^{-}(t)=I_{m}-j+i \Lambda(t, k)^{*} S(t, k)^{-1}\left(\alpha+i I_{n}\right)^{-1} \Lambda(t, k) j+ \\
& -i j \Lambda(t, k)^{*}\left(\alpha^{*}+i I_{n}\right)^{-1} S(t, k)^{-1} \Lambda(t, k)-\Lambda(t, k)^{*} S(t, k)^{-1} \times \\
& \times\left(\alpha+i I_{n}\right)^{-1} \Lambda(t, k) j \Lambda(t, k)^{*}\left(\alpha^{*}+i I_{n}\right)^{-1} S(t, k)^{-1} \Lambda(t, k) .
\end{aligned}
$$

It remains to show that $V_{k}^{+}(t)=H_{k}^{+}(t)$ and $V_{k}^{-}(t)=H_{k}^{-}(t)$. To do this note that (3.51) and (3.52) tell us that $H_{k}^{+}(t)$ and $H_{k}^{-}(t)$ are also given by

$$
\begin{aligned}
& H_{k}^{+}(t)=I_{m}+W_{\Sigma_{t, k}}(i) j W_{\Sigma_{t, k}}(-i)^{*}, \\
& H_{k}^{-}(t)=I_{m}-W_{\Sigma_{t, k}}(-i) j W_{\Sigma_{t, k}}(i)^{*} .
\end{aligned}
$$

Now use (4.47) and compute the products. This shows that $H_{k}^{+}(t)$ is equal to the right-hand side of (4.42) and $H_{k}^{-}(t)$ is equal to the right-hand side of (4.43). In other words, $V_{k}^{+}(t)=H_{k}^{+}(t)$ and $V_{k}^{-}(t)=H_{k}^{-}(t)$, and hence (4.38) is proved.

The FOURTH DERIVATIVE IN (4.31). In this part we shall show that

$$
\begin{aligned}
\left(\frac{d}{d t} W_{\Sigma_{t, k}}\right)(\lambda)=\left(\frac{H_{k}^{+}(t)}{\lambda-i}\right. & \left.+\frac{H_{k}^{-}(t)}{\lambda+i}\right) W_{\Sigma_{t, k}}(\lambda)+ \\
& -2 W_{\Sigma_{t, k}}(\lambda)\left(\frac{P_{1}}{\lambda-i}+\frac{P_{2}}{\lambda+i}\right) .
\end{aligned}
$$


First, recall that $W_{\Sigma_{t, k}}$ is given by

$$
W_{\Sigma_{t, k}}(z)=I_{m}+i \Lambda(t, k)^{*} S(t, k)^{-1}\left(z I_{n}-\alpha\right)^{-1} \Lambda(t, k) .
$$

Using (4.32) and (4.38), we obtain

$$
\begin{aligned}
\left(\frac{d}{d t} W_{\Sigma_{t, k}}\right)(\lambda)=i\left(H_{k}^{+}(t) \Lambda(t, k)^{*} S(t, k)^{-1}\left(\alpha-i I_{n}\right)^{-1}\left(\lambda I_{n}-\alpha\right)^{-1} \Lambda(t, k)+\right. \\
\quad+H_{k}^{-}(t) \Lambda(t, k)^{*} S(t, k)^{-1}\left(\alpha+i I_{n}\right)^{-1}\left(\lambda I_{n}-\alpha\right)^{-1} \Lambda(t, k)+ \\
\quad-2 \Lambda(t, k)^{*} S(t, k)^{-1}\left(\alpha-i I_{n}\right)^{-1}\left(\lambda I_{n}-\alpha\right)^{-1} \Lambda(t, k) P_{1}+ \\
\left.\quad-2 \Lambda(t, k)^{*} S(t, k)^{-1}\left(\alpha+i I_{n}\right)^{-1}\left(\lambda I_{n}-\alpha\right)^{-1} \Lambda(t, k) P_{2}\right) .
\end{aligned}
$$

Clearly we have

$$
\begin{aligned}
\left(\alpha-i I_{n}\right)^{-1}\left(\lambda I_{n}-\alpha\right)^{-1} & =(\lambda-i)^{-1}\left(\left(\alpha-i I_{n}\right)^{-1}+\left(\lambda I_{n}-\alpha\right)^{-1}\right) \\
\left(\alpha+i I_{n}\right)^{-1}\left(\lambda I_{n}-\alpha\right)^{-1} & =(\lambda+i)^{-1}\left(\left(\alpha+i I_{n}\right)^{-1}+\left(\lambda I_{n}-\alpha\right)^{-1}\right) .
\end{aligned}
$$

Taking into account (4.47), (4.49) and (4.50), we rewrite (4.48) as

$$
\begin{aligned}
\left(\frac{d}{d t} W_{\Sigma_{t, k}}\right) & (\lambda)=(\lambda-i)^{-1} H_{k}^{+}(t)\left(W_{\Sigma_{t, k}}(\lambda)-W_{\Sigma_{t, k}}(i)\right)+ \\
& +(\lambda+i)^{-1} H_{k}^{-}(t)\left(W_{\Sigma_{t, k}}(\lambda)-W_{\Sigma_{t, k}}(-i)\right)+ \\
& -2(\lambda-i)^{-1}\left(W_{\Sigma_{t, k}}(\lambda)-W_{\Sigma_{t, k}}(i)\right) P_{1} \\
& -2(\lambda+i)^{-1}\left(W_{\Sigma_{t, k}}(\lambda)-W_{\Sigma_{t, k}}(-i)\right) P_{2}
\end{aligned}
$$

Applying (3.46) and (3.47) with $\Sigma_{t, k}$ in place of $\Sigma_{k}$ we see that

$$
H_{k}^{+}(t) W_{\Sigma_{t, k}}(i)=2 W_{\Sigma_{t, k}}(i) P_{1}, \quad H_{k}^{-}(t) W_{\Sigma_{t, k}}(-i)=2 W_{\Sigma_{t, k}}(-i) P_{2} .
$$

In view of (4.52), formula (4.51) can be rewritten as (4.46).

Proof of Proposition 4.5. Let $Y$ be the matrix fucntion defined by (4.30). The fact that $Y_{k}(t, z)$ is a solution of equation (4.2) follows by applying Theorem 3.5 with $\Sigma_{t}$ in place of $\Sigma$. Similarly, using (4.46) and the fact that the matrices $P_{1}, P_{2}$, and $j$ are mutually commutative, we see that $Y_{k}(t, z)$ is 
a solution of the system (4.3). Indeed,

$$
\begin{aligned}
\frac{d}{d t} Y_{k}(t, z)= & \left(\frac{d}{d t} W_{\Sigma_{t, k}}\right)(-z)\left(I_{m}+\frac{i}{z} j\right)^{k} \exp \left\{-2 t\left(\frac{P_{1}}{z+i}+\frac{P_{2}}{z-i}\right)\right\}+ \\
& -2 Y_{k}(t, z)\left(\frac{P_{1}}{z+i}+\frac{P_{2}}{z-i}\right) \\
= & \left(\frac{H_{k}^{+}(t)}{-z-i}+\frac{H_{k}^{-}(t)}{-z+i}\right) Y_{k}(t, z)-2 Y_{k}(t, z)\left(\frac{P_{1}}{-z-i}+\frac{P_{2}}{-z+i}\right)+ \\
= & -\left(\frac{H_{k}^{+}(t)}{z+i}+\frac{H_{k}^{-}(t)}{z-i}\right) Y_{k}(t, z) .
\end{aligned}
$$

Thus $Y$ has the desired properties.

Remark 4.7. The equality $H_{k}^{-}=2 I_{m}-\left(H_{k}^{+}\right)^{*}$ is immediate from (4.44) and (4.45).

A remark on the continuous space variable $x$ analogue. The transfer matrix function of the form (2.10) depending on the continuous parameter $t$ and discrete parameter $k$, which we use in this section, appeared in [27], whereas the transfer matrix function (of the form (2.10)) depending on continuous parameters $x$ and $t$ appeared first in [36,37] (see also [22] and [23]). To explain the case of two continuous parameters in more detail we begin with the following analogue of Lemma 4.4.

Lemma 4.8. Let $\Sigma=\left\{\alpha, S_{0}, \vartheta_{1}, \vartheta_{2}\right\}$ be a strongly admissible quadruple, and let $k$ be a positive integer. Define

$$
\begin{aligned}
& \vartheta_{1}(t)=e^{-i t \alpha^{k}} \vartheta_{1}, \quad \vartheta_{2}(t)=e^{i t \alpha^{k}} \vartheta_{2}, \quad \Lambda(t)=\left[\begin{array}{ll}
\vartheta_{1}(t) & \vartheta_{2}(t)
\end{array}\right], \\
& S(t)=S_{0}+\int_{0}^{t} \sum_{\nu=1}^{k} \alpha^{\nu-1} \Lambda(s) j \Lambda(s)\left(\alpha^{*}\right)^{k-\nu} d s, \quad t \geq 0 .
\end{aligned}
$$

Then for each $t \in \mathbb{R}$ the quadruple $\Sigma(t)=\left\{\alpha, S(t), \vartheta_{1}(t), \vartheta_{2}(t)\right\}$ is strongly admissible.

The proof of the above lemma follows the same line of reasoning as used in the proof of Lemma 4.4. We omit the details. 
Next, using the above lemma, we construct a family of pseudo-exponential potentials depending continuously on an additional variable $t \in \mathbb{R}$. The starting point is the pseudo-exponential potential $v$ in (2.1) defined by the strongly admissible quadruple $\Sigma=\left\{\alpha, S_{0}, \vartheta_{1}, \vartheta_{2}\right\}$. Fix $t \in \mathbb{R}$, and let $\Sigma(t)$ be the strongly admissible quadruple defined in Lemma 4.8, i.e., $\Sigma(t)=$ $\left\{\alpha, S(t), \vartheta_{1}(t), \vartheta_{2}(t)\right\}$. In particular, $S(t)$ is given by (4.53). We apply Lemma 2.1 with $\Sigma(t)$ in place of $\Sigma$. Put

$$
\begin{aligned}
\vartheta_{1}(x, t) & =e^{-i x \alpha} \vartheta_{1}(t)=e^{-i\left(x \alpha+i t \alpha^{k}\right)} \vartheta_{1}, \\
\vartheta_{2}(x, t) & =e^{i x \alpha} \vartheta_{2}(t)=e^{i\left(x \alpha+i t \alpha^{k}\right)} \vartheta_{2}, \\
\Lambda(x, t) & =\left[\begin{array}{ll}
\vartheta_{1}(x, t) & \vartheta_{2}(x, t)
\end{array}\right] \\
S(x, t) & =S(t)+\int_{0}^{x} \Lambda(s, t) j \Lambda(s, t)^{*} d s, \quad x \geq 0 .
\end{aligned}
$$

Then, by Lemma 2.1, the quadruple

$$
\Sigma(x, t)=\left\{\alpha, S(x, t), \vartheta_{1}(x, t), \vartheta_{2}(x, t)\right\}
$$

is admissible for each $x \geq 0$. Furthermore, the corresponding pseudoexponential is given by

$$
v(x, t)=2 \vartheta_{1}^{*} e^{i\left(x \alpha^{*}+i t\left(\alpha^{*}\right)^{k}\right)} S(x, t)^{-1} e^{i\left(x \alpha+i t \alpha^{k}\right)} \vartheta_{2}, \quad x \geq 0 .
$$

In this way we obtain a family of pseudo-exponential potentials depending on the additional parameter $t$.

For $k=2,3$ the potential $v$ in (4.56) satisfies important integrable nonlinear equations; see, e.g., [36, Section 2] and [23, Section 4]. Namely, for $k=2$ the function $v$ is a solution of the matrix nonlinear Schrödinger equation

$$
2 \frac{\partial v}{\partial t}+i \frac{\partial^{2} v}{\partial x^{2}}+2 i v v^{*} v=0
$$

and for $k=3$ it satisfies the matrix modified Korteweg-de Vries equation

$$
4 \frac{\partial v}{\partial t}+\frac{\partial^{3} v}{\partial x^{3}}+3\left(\frac{\partial v}{\partial x} v^{*} v+v v^{*} \frac{\partial v}{\partial x}\right)=0
$$

The proofs of the above results can be obtained by direct computations as in [36, Section 2] and [23, Section 4] or by applying the generalized Bäcklund-Darboux transformation [38] to auxiliary linear systems and using 
compatibility condition (zero curvature equation) $G_{t}-F_{x}+G F-F G=0$, which is a continuous case equivalent of the compatibility condition (4.1). For instance, equation (4.57) is equivalent to the compatibility condition $G_{t}-F_{x}+G F-F G=0$, where $G=i z j+V(x, t)$,

$$
F=i\left(z^{2} j-i z j V(x, t)-\left(V_{x}(x, t)+j V(x, t)^{2}\right) / 2\right),
$$

and $V$ has the form (1.2).

\section{A Appendix: admissible quadruples}

Let $S_{0}$ and $\alpha$ be $n \times n$ matrices, and let $\vartheta_{1}$ and $\vartheta_{2}$ be matrices of sizes $n \times m_{1}$ and $n \times m_{2}$, respectively. The quadruple $\left\{\alpha, S_{0}, \vartheta_{1}, \vartheta_{2}\right\}$ is called admissible (see the beginning of Section 2) if

$$
S_{0}>0 \text { and } \alpha S_{0}-S_{0} \alpha^{*}=i \Lambda \Lambda^{*}, \text { where } \Lambda:=\left[\begin{array}{ll}
\vartheta_{1} & \vartheta_{2}
\end{array}\right] .
$$

By definition the transfer function associated with the quadruple

$$
\Sigma=\left\{\alpha, S_{0}, \vartheta_{1}, \vartheta_{2}\right\}
$$

is the matrix function $W_{\Sigma}$ given by (see (2.10) and (3.15)):

$$
W_{\Sigma}(z)=I_{m}+i \Lambda^{*} S_{0}^{-1}\left(z I_{n}-\alpha\right)^{-1} \Lambda, \text { where } m:=m_{1}+m_{2} .
$$

With $\Sigma$ we associate two other rational matrix functions:

$$
\begin{aligned}
& \varphi_{1, \Sigma}(z)=i \vartheta_{2}^{*} S_{0}^{-1}\left(z I_{n}-\beta_{1}\right)^{-1} \vartheta_{1}, \text { where } \beta_{1}:=\alpha-i \vartheta_{1} \vartheta_{1}^{*} S_{0}^{-1} \\
& \varphi_{2, \Sigma}(z)=-i \vartheta_{1}^{*} S_{0}^{-1}\left(z I_{n}+\beta_{2}\right)^{-1} \vartheta_{2}, \quad \text { where } \beta_{2}:=\alpha-i \vartheta_{2} \vartheta_{2}^{*} S_{0}^{-1} .
\end{aligned}
$$

If $\Sigma=\left\{\alpha, S_{0}, \vartheta_{1}, \vartheta_{2}\right\}$ is an admissible quadruple, then the same holds true for the associate quadruple

$$
\Sigma^{\#}:=\left\{\alpha, S_{0}, \vartheta_{2}, \vartheta_{1}\right\}
$$

and a direct computation shows that

$$
\varphi_{1, \Sigma \#}(-z)=\varphi_{2, \Sigma}(z) .
$$


Admissible quadruples are closely related to symmetric S-nodes (see [44, Section 2.2]). Indeed, if $\Sigma=\left\{\alpha, S_{0}, \vartheta_{1}, \vartheta_{2}\right\}$ is an admissible quadruple, then the matrices

$$
A:=\alpha, \quad S:=S_{0}, \quad \Pi_{1}:=\Lambda, \quad \Gamma_{1}:=S_{0}^{-1} \Lambda,
$$

form a symmetric S-node (with $J=I_{n}$ ) as defined in Section 2.2 of [44]. Using this connection, it is readily seen that item (iii) in the following lemma is a special case of Theorem 2.2.1 in [44] (see also [42], Theorem 17.1 in [7] or Corollary 1.15 in [41]).

Lemma A.1. Let $\Sigma=\left\{\alpha, S_{0}, \vartheta_{1}, \vartheta_{2}\right\}$ be an admissible quadruple. Then

(i) $\sigma(\alpha) \subset \overline{\mathbb{C}_{+}}$;

(ii) if, in addition, the pair $\{\alpha, \Lambda\}$ is controllable, then $\sigma(\alpha) \subset \mathbb{C}_{+}$, and in that case $S_{0}$ is uniquely determined by $\alpha$ and $\Lambda$. More precisely, $S_{0}$ is given by

$$
S_{0}=\frac{1}{2 \pi} \int_{-\infty}^{\infty}\left(\lambda I_{n}-\alpha\right)^{-1} \Lambda \Lambda^{*}\left(\lambda I_{n}-\alpha^{*}\right)^{-1} d \lambda
$$

(iii) $W_{\Sigma}$ is a rational Schur class function and its values on the real line are unitary matrices. Moreover, we have

$$
\begin{aligned}
I_{m}- & W_{\Sigma}(z) W_{\Sigma}(\bar{\zeta})^{*}= \\
\quad & =i(z-\zeta) \Lambda^{*} S_{0}^{-1}\left(z I_{n}-\alpha\right)^{-1} S_{0}\left(\zeta I_{n}-\alpha\right)^{-1} S_{0}^{-1} \Lambda \\
I_{m}- & W_{\Sigma}(z)^{*} W_{\Sigma}(z)=i(z-\bar{z}) \Lambda^{*}\left(\bar{z} I_{n}-\alpha^{*}\right)^{-1} S_{0}^{-1}\left(z I_{n}-\alpha\right)^{-1} \Lambda .
\end{aligned}
$$

Proof. In remains to prove items (i) and (ii). Since $S_{0}$ is positive definite, the second part of (A.1) implies that

$$
\left(S_{0}^{-1 / 2} \alpha S_{0}^{1 / 2}\right)-\left(S_{0}^{-1 / 2} \alpha S_{0}^{-1 / 2}\right)^{*}=i S_{0}^{-1 / 2} \Lambda \Lambda^{*} S_{0}^{-1 / 2}, \quad S_{0}^{-1 / 2} \Lambda \Lambda^{*} S_{0}^{-1 / 2} \geq 0
$$

It follows that the numerical range of the matrix $S_{0}^{-1 / 2} \alpha S_{0}^{1 / 2}$ is a subset of $\overline{\mathbb{C}_{+}}$, and hence $\sigma(\alpha)=\sigma\left(S_{0}^{-1 / 2} \alpha S_{0}^{1 / 2}\right) \subset \overline{\mathbb{C}_{+}}$. This proves item (i).

The first statement in item (ii) is a straightforward application of the classical Chen-Wimmer inertia theorem (see Corollary 1 in [33, Section 13.1]). For (A.7) see [26] or Theorem I.4.1 in [21] (see, also (3.10)). 
Lemma A.2. Let $\Sigma=\left\{\alpha, S_{0}, \vartheta_{1}, \vartheta_{2}\right\}$ be an admissible quadruple, and partition its transfer function $W_{\Sigma}$ as a $2 \times 2$ block matrix function,

$$
W_{\Sigma}(z)=\left[\begin{array}{ll}
a(z) & b(z) \\
c(z) & d(z)
\end{array}\right] \text {, where } a(z) \text { has size } m_{1} \times m_{1} .
$$

Then the associated functions $\varphi_{1, \Sigma}$ and $\varphi_{2, \Sigma}$ are given by

$$
\varphi_{1, \Sigma}(z)=c(z) a(z)^{-1} \quad \text { and } \quad \varphi_{2, \Sigma}(z)=b(-z) d(-z)^{-1} .
$$

Proof. From (A.2) and $\Lambda=\left[\begin{array}{ll}\vartheta_{1} & \vartheta_{2}\end{array}\right]$ it follows that

$$
a(z)=I_{m_{1}}+i \vartheta_{1}^{*} S_{0}^{-1}\left(z I_{n}-\alpha\right)^{-1} \vartheta_{1}, \quad c(z)=i \vartheta_{2}^{*} S_{0}^{-1}\left(z I_{n}-\alpha\right)^{-1} \vartheta_{1} .
$$

Since $a$ is a proper rational matrix function whose value at infinity is the $m_{1} \times m_{1}$ identity matrix, a classical inversion theorem (see, e.g., Theorem 2.2 in [6]) tells us that

$$
a(z)^{-1}=I_{m_{1}}-i \vartheta_{1}^{*} S_{0}^{-1}\left(z I_{n}-\alpha^{\times}\right)^{-1} \vartheta_{1}, \text { where } \alpha^{\times}=\alpha-i \vartheta_{1} \vartheta_{1}^{*} S_{0}^{-1}=\beta_{1} .
$$

Hence, again using Theorem 2.2 in [6] and taking into account (A.3), we know that

$$
\begin{aligned}
c(z) a(z)^{-1} & =i \vartheta_{2}^{*} S_{0}^{-1}\left(z I_{n}-\alpha\right)^{-1} \vartheta_{1} a(z)^{-1}=i \vartheta_{2}^{*} S_{0}^{-1}\left(z I_{n}-\alpha^{\times}\right)^{-1} \vartheta_{1} \\
& =i \vartheta_{2}^{*} S_{0}^{-1}\left(z I_{n}-\beta_{1}\right)^{-1} \vartheta_{1}=\varphi_{1, \Sigma}(z),
\end{aligned}
$$

which proves the first part of (A.11).

To prove the second identity in (A.11) we use the associate admissible quadruple $\Sigma^{\#}=\left\{\alpha, S_{0}, \vartheta_{2}, \vartheta_{1}\right\}$ and equality (A.6). Note that

$$
\left[\begin{array}{ll}
\vartheta_{2} & \vartheta_{1}
\end{array}\right]=\left[\begin{array}{ll}
\vartheta_{1} & \vartheta_{2}
\end{array}\right] U, \text { where } U=\left[\begin{array}{cc}
0 & I_{m_{1}} \\
I_{m_{2}} & 0
\end{array}\right]
$$

Since $U$ is unitary, it is clear from (A.12) and (A.2) that $W_{\Sigma \#}=U^{*} W_{\Sigma} U$. But then, using the special form of $U$, we obtain

$$
W_{\Sigma \#}=U^{*} W_{\Sigma} U=\left[\begin{array}{ll}
d(z) & c(z) \\
b(z) & a(z)
\end{array}\right] .
$$

Applying the result of the first part of the proof to $\Sigma^{\#}$ in place of $\Sigma$ we see that $\varphi_{1, \Sigma^{\#}}=b(z) d(z)^{-1}$. But then (A.6) yields the second identity in (A.11). 
Theorem A.3. Let $\varphi$ be an $m_{2} \times m_{1}$ rational matrix function. Then $\varphi$ coincides with some function $\varphi_{1, \Sigma}$ associated with an admissible quadruple $\Sigma$ if and only if $\varphi$ is strictly proper. In that case, the corresponding $\Sigma$ can be obtained explicitly by using the following procedure.

Step 1. Let $n$ be the McMillan degree of $\varphi$, and construct a minimal realization of $\varphi$ :

$$
\varphi(z)=i \theta_{2}^{*}\left(z I_{n}-\gamma\right)^{-1} \theta_{1} .
$$

Step 2. Choose $X$ to be the unique positive definite solution of the algebraic Riccati equation

$$
\gamma X-X \gamma^{*}-i X \theta_{2} \theta_{2}^{*} X+i \theta_{1} \theta_{1}^{*}=0 .
$$

Step 3. Put

$$
\begin{gathered}
S_{0}=I_{n}, \quad \vartheta_{1}=X^{-1 / 2} \theta_{1}, \quad \vartheta_{2}=X^{1 / 2} \theta_{2}, \\
\alpha=X^{-1 / 2} \gamma X^{1 / 2}+i \vartheta_{1} \vartheta_{1}^{*} .
\end{gathered}
$$

With these choices the quadruple $\Sigma=\left\{\alpha, S_{0}, \vartheta_{1}, \vartheta_{2}\right\}$ is admissible and $\varphi$ coincides with its first associate function $\varphi_{1, \Sigma}$. Moreover, in this case the pairs $\left\{\alpha, \vartheta_{1}\right\}$ and $\left\{\alpha, \vartheta_{2}\right\}$ are controllable.

Proof. First note that the minimality of the realization (A.14) is equivalent to the requirement that simultaneously the pair $\left\{\theta_{2}^{*}, \gamma\right\}$ is observable and the pair $\left\{\gamma, \theta_{1}\right\}$ is controllable. But then we can apply Proposition 2.2 in [23] to show that the Riccati equation (A.15) has a positive definite solution $X$, a result which has its roots in Kalman's theory of mathematical systems [28] (see also [32, pp. 358 and 369], where the uniqueness of $X$ is shown as well). The remaining part of the proof is split into two parts.

PART 1. Using the definition of $\alpha$ in (A.17), the unique positive definite solution $X$ of the Riccati equation (A.15), and the definitions of $\vartheta_{1}$ and $\vartheta_{2}$ in (A.16) we see that

$$
\begin{aligned}
\alpha-\alpha^{*} & =X^{-1 / 2} \gamma X^{1 / 2}-X^{1 / 2} \gamma^{*} X^{-1 / 2}+2 i \vartheta_{1} \vartheta_{1}^{*} \\
& =i X^{1 / 2} \theta_{2} \theta_{2}^{*} X^{1 / 2}-i X^{-1 / 2} \theta_{1} \theta_{1}^{*} X^{-1 / 2}+2 i \vartheta_{1} \vartheta_{1}^{*} \\
& =i \vartheta_{2} \vartheta_{2}^{*}-i \vartheta_{1} \vartheta_{1}^{*}+2 i \vartheta_{1} \vartheta_{1}^{*}=i \vartheta_{2} \vartheta_{2}^{*}+i \vartheta_{1} \vartheta_{1}^{*} .
\end{aligned}
$$


Since $S_{0}=I_{n}$ by definition, we conclude that $\Sigma=\left\{\alpha, S_{0}, \vartheta_{1}, \vartheta_{2}\right\}$ is an admissible quadruple. Moreover, the associate function $\varphi_{1, \Sigma}$ is equal to $\varphi$. Indeed, in this case using (A.17), we have

$$
\beta_{1}=\alpha-i \vartheta_{1} \vartheta_{1}^{*}=X^{-1 / 2} \gamma X^{1 / 2} \text {. }
$$

Thus $X^{1 / 2} \beta_{1} X^{-1 / 2}=\gamma$, and hence

$$
\begin{aligned}
\varphi_{1, \Sigma}(z) & =i \vartheta_{2}^{*} S_{0}^{-1}\left(z I_{n}-\beta_{1}\right)^{-1} \vartheta_{1} \\
& =i \theta_{2}^{*} X^{1 / 2}\left(z I_{n}-\beta_{1}\right)^{-1} X^{-1 / 2} \theta_{1} \\
& =i \theta_{2}^{*}\left(z I_{n}-X^{1 / 2} \beta_{1} X^{-1 / 2}\right)^{-1} \theta_{1} \\
& =i \theta_{2}^{*}\left(z I_{n}-\gamma\right)^{-1} \theta_{1}=\varphi .
\end{aligned}
$$

PART 2. In this part we prove that the pairs $\left\{\alpha, \vartheta_{1}\right\}$ and $\left\{\alpha, \vartheta_{2}\right\}$ are controllable. Since the realization (A.14) is minimal and $\varphi=\varphi_{1, \Sigma}$, the same is valid for the realization (A.3), and hence the pairs $\left\{\beta_{1}, \vartheta_{1}\right\}$ and $\left\{\left(\beta_{1}\right)^{*}, \vartheta_{2}\right\}$ are controllable. Formula (A.3) implies that $\alpha=\beta_{1}+i \vartheta_{1} \vartheta_{1}^{*}$. Using the latter identity, the fact that $\left\{\beta_{1}, \vartheta_{1}\right\}$ is controllable and the equality $\operatorname{Im} \vartheta_{1}=\operatorname{Im} \vartheta_{1} \vartheta_{1}^{*}$, we conclude that $\left\{\alpha, \vartheta_{1}\right\}$ is controllable too.

It remains to prove that $\left\{\alpha, \vartheta_{2}\right\}$ is controllable. Since $S_{0}=I_{n}$ and the quadruple $\Sigma=\left\{\alpha, S_{0}, \vartheta_{1}, \vartheta_{2}\right\}$ is admissible, we have $\alpha-\alpha^{*}=i\left(\vartheta_{1} \vartheta_{1}^{*}+\vartheta_{2} \vartheta_{2}^{*}\right)$, and therefore

$$
\beta_{1}=\alpha-i \vartheta_{1} \vartheta_{1}^{*}=\alpha^{*}+i \vartheta_{2} \vartheta_{2}^{*}=\left(\alpha-i \vartheta_{2} \vartheta_{2}^{*}\right)^{*} .
$$

Thus $\alpha=\left(\beta_{1}\right)^{*}+i \vartheta_{2} \vartheta_{2}^{*}$. We know that $\left\{\left(\beta_{1}\right)^{*}, \vartheta_{2}\right\}$ is controllable and that $\operatorname{Im} \vartheta_{2}=\operatorname{Im} \vartheta_{2} \vartheta_{2}^{*}$. These facts imply that $\left\{\alpha, \vartheta_{2}\right\}$ is also controllable.

Using the identity (A.6) and Theorem A.3 we can show that a proper rational matrix function $\varphi$ also coincides with the second associate function $\varphi_{2, \Sigma}$ of some admissible quadruple $\Sigma$. More precisely, we have the following corollary.

Corollary A.4. Let $\widetilde{\varphi}$ be an $m_{2} \times m_{1}$ rational matrix function. Then $\widetilde{\varphi}$ coincides with some function $\varphi_{2, \Sigma}$ associated with an admissible quadruple $\Sigma$ if and only if $\varphi$ is strictly proper. The corresponding $\Sigma=\left\{\alpha, S_{0}, \vartheta_{1}, \vartheta_{2}\right\}$ can 
be obtained explicitly by using the following procedure. First, we construct a minimal realization of $\widetilde{\varphi}$,

$$
\widetilde{\varphi}(z)=-i \theta_{1}^{*}\left(z I_{n}+\gamma\right)^{-1} \theta_{2} .
$$

Next, we choose $X$ to be the unique positive definite solution of the Riccati equation $\gamma X-X \gamma^{*}-i X \theta_{1} \theta_{1}^{*} X+i \theta_{2} \theta_{2}^{*}=0$. Finally, we put

$$
\begin{gathered}
S_{0}=I_{n}, \quad \vartheta_{1}=X^{1 / 2} \theta_{1}, \quad \vartheta_{2}=X^{-1 / 2} \theta_{2}, \\
\alpha=X^{-1 / 2} \gamma X^{1 / 2}+i \vartheta_{2} \vartheta_{2}^{*} .
\end{gathered}
$$

Then $\widetilde{\varphi}=\varphi_{2, \Sigma}$ and the pairs $\left\{\alpha, \vartheta_{1}\right\}$ and $\left\{\alpha, \vartheta_{2}\right\}$ are controllable.

Proof. Put $\varphi(z)=\widetilde{\varphi}(-z)$. Then $\varphi$ is rational, and $\widetilde{\varphi}$ is strictly proper if and only if $\varphi$ is strictly proper. But, by Theorem $\underset{\widetilde{\Sigma}}{\text { A.3, the latter happens }}$ if and only if there exists an admissible quadruple $\widetilde{\Sigma}=\left\{\alpha, S_{0}, \widetilde{\vartheta}_{1}, \widetilde{\vartheta}_{2}\right\}$ such that $\varphi(z)=\varphi_{1, \widetilde{\Sigma}}(z)$. Next, taking into account (A.5), we see that $\widetilde{\Sigma}$ is the associate quadruple $\Sigma^{\#}$ for $\Sigma=\left\{\alpha, S_{0}, \widetilde{\vartheta}_{2}, \widetilde{\vartheta}_{1}\right\}$. Then we know from (A.6) that

$$
\widetilde{\varphi}(z)=\varphi(-z)=\varphi_{1, \Sigma \#}(-z)=\varphi_{2, \Sigma}(z) .
$$

Moreover, Theorem A.3 provides a method to construct $\widetilde{\Sigma}$, which in terms of $\widetilde{\Sigma}=\Sigma^{\#}$ (or, equivalently, $\Sigma=\widetilde{\Sigma}^{\#}$ ) yields the procedure to recover $\Sigma$ from $\widetilde{\varphi}$ and formula (A.4).

Proof of Proposition 2.8. Let $S(x)$ be the $n \times n$ matrix defined by (2.2), and put

$$
Q(x)=\left[\begin{array}{ll}
S_{0} & -i I_{n}
\end{array}\right] e^{-2 i x A}\left[\begin{array}{c}
I_{n} \\
0
\end{array}\right], \quad x \in \mathbb{R}_{+},
$$

where $A$ is given by (2.23). In order to prove Proposition 2.8 , that is, to prove (2.24), it suffices to show that $Q(x)=\Delta(x)$, where $\Delta(x)=e^{-i x \alpha} S(x) e^{-i x \alpha^{*}}$, for each $x \in \mathbb{R}_{+}$. For that we directly differentiate $\Delta(x)$ using the first identity in (2.7) (where we substitute the expression for $\Lambda$ from (2.2)) and obtain

$$
\frac{d}{d x} \Delta(x)=-i\left(\alpha \Delta(x)+\Delta(x) \alpha^{*}\right)+e^{-2 i x \alpha} \vartheta_{1} \vartheta_{1}^{*}-\vartheta_{2} \vartheta_{2}^{*} e^{-2 i x \alpha^{*}} .
$$


Now, we show that $Q$ satisfies the same first order linear differential equation

$$
\frac{d}{d x} Q(x)=-i\left(a Q(x)+Q(x) \alpha^{*}\right)+e^{-2 i x \alpha} \vartheta_{1} \vartheta_{1}^{*}-\vartheta_{2} \vartheta_{2}^{*} e^{-2 i x \alpha^{*}} .
$$

To prove (A.22) note that

$$
i \frac{d}{d x} Q(x)=\left[\begin{array}{ll}
S_{0} & -i I_{n}
\end{array}\right]\left(A e^{-2 i x A}+e^{-2 i x A} A\right)\left[\begin{array}{c}
I_{n} \\
0
\end{array}\right]
$$

Using (2.3) and the definition of $A$ in (2.23) we see that

$$
\begin{aligned}
{\left[\begin{array}{ll}
S_{0} & -i I_{n}
\end{array}\right] A } & =\left[\begin{array}{ll}
S_{0} \alpha^{*}+i \vartheta_{1} \vartheta_{1}^{*} & -i \alpha
\end{array}\right] \\
& =\left[\begin{array}{lll}
\alpha S_{0}-i \vartheta_{2} \vartheta_{2}^{*} & -i \alpha
\end{array}\right] \\
& =\alpha\left[\begin{array}{lll}
S_{0} & -i I_{n}
\end{array}\right]-i\left[\begin{array}{lll}
\vartheta_{2} \vartheta_{2}^{*} & 0
\end{array}\right]
\end{aligned}
$$

Furthermore, we have

$$
A\left[\begin{array}{c}
I_{n} \\
0
\end{array}\right]=\left[\begin{array}{c}
\alpha^{*} \\
-\vartheta_{1} \vartheta_{1}^{*}
\end{array}\right]=\left[\begin{array}{c}
I_{n} \\
0
\end{array}\right] \alpha^{*}+\left[\begin{array}{c}
0 \\
I_{n}
\end{array}\right]\left(-\vartheta_{1} \vartheta_{1}^{*}\right) .
$$

It follows (using again the definiton of $A$ in (2.23)) that

$$
\begin{gathered}
i \frac{d}{d x} Q(x)=\alpha\left[\begin{array}{ll}
S_{0} & -i I_{n}
\end{array}\right] e^{-2 i x A}\left[\begin{array}{c}
I_{n} \\
0
\end{array}\right]-i\left[\begin{array}{ll}
\vartheta_{2} \vartheta_{2}^{*} & 0
\end{array}\right] e^{-2 i x A}\left[\begin{array}{c}
I_{n} \\
0
\end{array}\right] \\
+\left[\begin{array}{ll}
S_{0} & -i I_{n}
\end{array}\right] e^{-2 i x A}\left[\begin{array}{c}
I_{n} \\
0
\end{array}\right] \alpha^{*} \\
+\left[\begin{array}{ll}
S_{0} & -i I_{n}
\end{array}\right] e^{-2 i x A}\left[\begin{array}{c}
0 \\
I_{n}
\end{array}\right]\left(-\vartheta_{1} \vartheta_{1}^{*}\right) \\
=\alpha Q(x)-i \vartheta_{2} \vartheta_{2}^{*} e^{-2 i x \alpha^{*}}+Q(x) \alpha^{*}+i e^{-2 i x \alpha} \vartheta_{1} \vartheta_{1}^{*} .
\end{gathered}
$$

Equation (A.22) is immediate from $Q$. Since $\Delta$ and $Q$ satisfy the same first order linear differential equation with the same initial condition $\Delta(0)=$ $Q(0)=S_{0}$, we see that $\Delta(x)=Q(x)$ for each $x \in \mathbb{R}_{+}$, as desired.

Acknowledgments. The research of A.L. Sakhnovich was supported by the Austrian Science Fund (FWF) under Grant No. P24301. The authors are grateful to I. Roitberg for her help and useful remarks. 


\section{References}

[1] D. Alpay and I. Gohberg. Inverse spectral problems for difference operators with rational scattering matrix function. Integral Equations Operator Theory, 20:125-170, 1994.

[2] D. Alpay and I. Gohberg. Inverse spectral problem for differential operators with rational scattering matrix functions. J. Differential Equations, 118:1-19, 1995.

[3] D. Alpay, I. Gohberg, M.A. Kahshoek, L. Lerer, A. SAKHNOVICH, Krein systems and canonical systems on a finite interval: accelerants with a jump discontinuity at the origin and continuous potentials, Integral Equations Operator Theory 68:1 (2010) $115-150$.

[4] A.V. Bäcklund. Zur Theorie der partiellen Differentialgleichungen erster Ordnung. Math. Ann., 17:285-328, 1880.

[5] H. Bart, I. Gohberg and M. A. Kaashoek. Minimal factorization of matrix and operator functions. Oper. Theory Adv. Appl. 1. Basel/Boston: Birkhäuser, 227 p., 1979.

[6] H. Bart, I. Gohberg, M. A. Kaashoek, A.C.M. Ran, Factorization of matrix and operator functions: the state space method, Oper. Theory Adv. Appl. 178, Birkhäuser, Basel-Boston, 2008.

[7] H. Bart, I. Gohberg, M. A. Kaashoek, A.C.M. Ran, A state space approach to canonical factorization with applications, Oper. Theory Adv. Appl. 200, Birkhäuser, Basel-Boston, 2010.

[8] S. Clark, F. Gesztesy, On self-adjoint and J-self-adjoint Diractype Operators: A Case Study, Contemp. Math. 412 (2006) 103-140.

[9] M. M. Crum, Associated Sturm-Liouville systems, Quart. J. Math. Oxford (2) 6 (1955) 121-127.

[10] G. Darboux. Sur les quations aux drives partielles du second ordre. Ann. de l'c. Norm., VII:163-173, 1870. 
[11] G. Darboux. Lecons sur la Theorie Generale de Surface et les Applications Geometriques du Calcul Infinitesimal. II: Les congruences et les quations linaires aux drives partielles. Des lignes traces sur les surfaces. Paris: Gauthier-Villars \& Fils, 1889.

[12] P.A. DeIfT, Applications of a commutation formula, Duke Math. J. 45 (1978) 267-310.

[13] Dimakis, Aristophanes(GR-AEG3-FNB); Mller-Hoissen, Folkert(DMPI-DYO) Bidifferential calculus approach to AKNS hierarchies and their solutions. (English summary) SIGMA Symmetry Integrability Geom. Methods Appl. 6 (2010), Paper 055, 27 pp.

[14] L.D. Faddeev, L.A. Takhtajan, Hamiltonian methods in the theory of solitons, Springer-Verlag, NY, 1986.

[15] B. Fritzsche, B. Kirstein, I.Ya. Roitberg, A.L. SAKHNOVICH, Weyl matrix functions and inverse problems for discrete Dirac type selfadjoint system: explicit and general solutions, Operators and Matrices 2 (2008) 201-231.

[16] B. Fritzsche, B. Kirstein, I.Ya. Roitberg, and A.L. Sakhnovich, Skew-Self-Adjoint Dirac System with a Rectangular Matrix Potential: Weyl Theory, Direct and Inverse Problems, Integral Equations and Operator Theory 74:2 (2012), 163-187.

[17] B. Fritzsche, B. Kirstein, I.Ya. Roitberg and A.L. Sakhnovich. Weyl theory and explicit solutions of direct and inverse problems for Dirac system with a rectangular matrix potential. Oper. Matrices, 7(1):183-196, 2013.

[18] B. Fritzsche, B. Kirstein, I.Ya. Roitberg, and A.L. Sakhnovich, Discrete Dirac system: rectangular Weyl functions, direct and inverse problems, Oper. Matrices, 8(3):799-819, 2014.

[19] F. Gesztesy, A complete spectral characterization of the double commutation method, J. Funct. Anal. 117 (1993) 401-446.

[20] F. Gesztesy, G. Teschl, On the double commutation method, Proc. Amer. Math. Soc. 124 (1996) 1831-1840. 
[21] I. Gohberg, S. Goldberg, and M.A. Kaashoek: Classes of Linear Operators, Volume I. Oper. Theory Adv. Appl. 49, Birkhäuser Verlag, Basel (1990)

[22] I. Gohberg, M.A.Kaashoek and A.L.Sakhnovich: Canonical systems with rational spectral densities: explicit formulas and applications, Mathematische Nachr. 194 (1998), 93-125.

[23] I.Gohberg, M.A.Kaashoek and A.L.Sakhnovich: Pseudocanonical systems with rational Weyl functions: explicit formulas and applications, Journal Diff. Eq. 146 (1998), 375-398.

[24] I. Gohberg, M.A. Kanshoek, A.L. Sakhnovich, Scattering problems for a canonical system with a pseudo-exponential potential, Asymptotic Analysis 29:1 (2002) 1-38.

[25] Golubchik I.Z., Sokolov V.V., Generalized Heisenberg equations on Z-graded Lie algebras, Theoret. and Math. Phys. 120 (1999), 10191025 .

[26] Ivanchenko, T. S., Sakhnovich, L. A., An operator approach to V. P. Potapov's scheme for the investigation of interpolation problems. Ukrainian Math. J. 39 (1987), no. 5, 464-469.

[27] M.A. Kaashoek and A.L. Sakhnovich. Discrete skew selfadjoint canonical system and the isotropic Heisenberg magnet model. $J$. Funct. Anal., 228:207-233, 2005.

[28] R.E. Kalman, P. Falb, M. Arbib, Topics in mathematical system theory, McGraw-Hill, New York, 1969.

[29] P. P. Kulish and N. Yu. Reshetikhin, The generalized Heisenberg ferromagnet and the Gross-Neveu model, Soviet Phys. JETP 80 (1981), no. 1, 214-228

[30] M.G. KREIn, Continuous analogues of propositions on polynomials orthogonal on the unit circle (Russian), Dokl. Akad. Nauk SSSR 105 (1955) 637-640.

[31] M.G. KREIN, On a continual analogue of a Christoffel formula from the theory of orthogonal polynomials (Russian), Dokl. Akad. Nauk SSSR (N.S.) 113 (1957) 970-973. 
[32] P. Lancaster, L. Rodman, Algebraic Riccati equations, Clarendon Press, Oxford, 1995.

[33] P. Lancaster and M. Tismenetsky, The theory of matrices, Academic Press, INC., Boston, 1985.

[34] B.M. Levitan, I.S. SARgSJan, Sturm-Liouville and Dirac operators, Mathematics and its Applications 59, Kluwer, Dordrecht, 1990 .

[35] A.L. Sakhnovich, A nonlinear Schrödinger equation on the semiaxis and a related inverse problem, Ukrain. Math. J. 42:3 (1990) 316-323.

[36] A.L. Sakhnovich: Exact solutions of nonlinear equations and the method of operator identities, Lin. Alg. Appl. 182 (1993), 109-126.

[37] A.L. Sakhnovich: Dressing procedure for solutions of nonlinear equations and the method of operator identities, Inverse problems 10 (1994), 699-710.

[38] A.L. Sakhnovich: Generalized Bäcklund-Darboux transformation: spectral properties and nonlinear equations, Journal Math. Analysis and Applications 262:1 (2001), 274-306.

[39] A.L. Sakhnovich, Dirac type and canonical systems: spectral and Weyl-Titchmarsh functions, direct and inverse problems, Inverse Problems 18 (2002) 331-348.

[40] A.L. Sakhnovich: Skew-selfadjoint discrete and continuous Diractype systems: inverse problems and Borg-Marchenko theorems, Inverse Problems 22 (2006), 2083-2101.

[41] Sakhnovich A. L., Sakhnovich L. A. and Roitberg I. Ya. Inverse problems and nonlinear evolution equations. Solutions, Darboux matrices and Weyl-Titchmarsh functions de Gruyter Studies in Mathematics vol 47, Berlin: De Gruyter, 2013.

[42] L.A. Sakhnovich, On the factorization of the transfer matrix function, Sov. Math. Dokl. 17 (1976) 203-207. 
[43] L.A. Sakhnovich, Factorisation problems and operator identities, Russian Math. Surv. 41 (1986) 1-64.

[44] L.A. Sakhnovich, Spectral theory of canonical differential systems. Method of operator identities, Oper. Theory Adv. Appl. 107, Birkhäuser, Basel-Boston, 1999.

[45] L.A. Sakhnovich. On Krein's differential system and its generalization. Integral Equations Operator Theory, 55: 561-572, 2006.

[46] Saleem, U.; Hassan, M. Quasideterminant solutions of the generalized Heisenberg magnet model. J. Phys. A 43 (2010), no. 4, 045204, $12 \mathrm{pp}$.

[47] E.K. Sklyanin, Some algebraic structures connected with the YangBaxter equation, Funct. Anal. Appl. 16 (1983), 263-270.

[48] Skrypnyk, T. Deformations of loop algebras and integrable systems: hierarchies of integrable equations. J. Math. Phys. 45 (2004), no. 12, 4578-4595.

[49] B. Sutherland, Model for a multicomponent quantum system, Phys. Rev. B 12, 3795-3805 (1975). 